\title{
Hydrodynamical models and synthetic spectra of circumstellar dust shells around AGB stars $^{\star}$
}

\section{Stationary solutions}

\author{
M. Steffen ${ }^{1,2}$, R. Szczerba ${ }^{3,4}$, A. Men'shchikov ${ }^{5}$, and D. Schönberner ${ }^{6}$ \\ 1 Astrophysikalisches Institut Potsdam, D-14473 Potsdam, Germany \\ e-mail: MSteffen@aip.de \\ 2 Institut für Astronomie und Astrophysik der Universität Kiel, D-24098 Kiel, Germany \\ e-mail: supas048@astrophysik.uni-kiel.de \\ 3 Nicolaus Copernicus Astronomical Center, PL-87-100 Torun, Poland \\ e-mail: szczerba@ncac.torun.pl \\ 4 Department of Physics and Astronomy, University of Calgary, Calgary, AB T2N 1N4, Canada \\ 5 Max-Planck-Gesellschaft, AG "Dust in star forming regions", D-07745 Jena, Germany \\ e-mail: sascha@georg.astro.uni-jena.de \\ ${ }^{6}$ Astrophysikalisches Institut Potsdam, D-14473 Potsdam, Germany \\ e-mail: Deschoenberner@aip.de
}

Received December 9, 1996; accepted February 5, 1997

\begin{abstract}
We present a sample of hydrodynamical steady state models of circumstellar gas/dust shells around late type giants together with computed spectral energy distributions (SEDs). In these models, the stellar wind is driven by radiation pressure on dust grains and subsequent momentum transfer to the gas molecules via collisions. Given the fundamental stellar parameters $\left(M_{*}\right.$, $\left.L_{*}, T_{\text {eff }}\right)$, the mass loss rate $(\dot{M})$, and the dust properties, a self-consistent physical model of the circumstellar gas/dust shell is obtained from the numerical solution of the coupled equations of hydrodynamics and radiative transfer. The computed outflow velocities and infrared fluxes of the circumstellar envelopes can be compared directly with the observed properties of stars on asymptotic giant branch. Plotting the positions of our steady state models in different IRAS two-color-diagrams, we confirm that, for fixed dust properties, all models fall on a simple color-color relation with $\dot{M}$ (or optical depth) as the only parameter. Surprisingly, we find a good agreement between the synthetic spectra resulting from the selfconsistent hydrodynamical approach and those obtained from much simpler models based on a constant outflow velocity and ignoring drift of dust relative to the gas.

Our models are compared with the results of similar calculations by Netzer \& Elitzur (1993). We find signif-

Send offprint requests to: M. Steffen

* Tables 2 to 11 are only available in electronic form at the CDS via anonymous ftp to cdsarc.u-strasbg.fr (130.79.128.5) or via http://cdsweb.u-strasbg.fr/Abstract.html
\end{abstract}

icant differences which are probably the result of some unrealistic approximations in the treatment of radiative transfer underlying the model calculations of Netzer \& Elitzur. Moreover, our results demonstrate that, in general, gas pressure cannot be neglected for winds with relatively low expansion velocities $\left(u_{\mathrm{e}}<30 \mathrm{~km} / \mathrm{s}\right)$. For given stellar parameters and dust properties, the theoretical minimum (maximum) mass loss rate decreases (increases) significantly when gas pressure is taken into account.

Key words: stars: AGB and post-AGB - circumstellar matter - stars: mass loss — dust, extinction hydrodynamics - radiative transfer

\section{Introduction}

Before turning into planetary nebulae, low to intermediate mass stars $\left(1-8 M_{\odot}\right)$ are found to evolve along the Asymptotic Giant Branch (AGB). In this phase the time scales of evolution are determined mainly by mass loss since this process dominates over nuclear burning. High mass loss rates between $10^{-6}$ and $10^{-4} M_{\odot} / \mathrm{yr}$ are very often detected, mostly by excess continuum radiation in the infrared, originating from thermal emission of circumstellar dust, and by the presence of molecular rotation lines from different molecules seen in emission at submm, $\mathrm{mm}$, and $\mathrm{cm}$ wavelengths. 
It is now widely accepted that the mechanism responsible for such high mass loss rates during the AGB evolution is based on the efficiency of radiation pressure on dust grains. Pushed away by shocks generated by the Mira-type stellar pulsations, the outflowing gas cools down until, at some distance from the star, heavy elements can condense. The dust grains belong to one of two different types: (1) silicate-type grains, found around so called "Oxygen stars" with an abundance ratio $\mathrm{C} / \mathrm{O}<1$, and (2) carbon-based grains around "Carbon stars" with $\mathrm{C} / \mathrm{O}>1$. The newly formed dust particles scatter and absorb stellar photons, in this way extracting momentum and energy from the radiation field. The absorbed stellar light is reemitted in the infrared while the acquired momentum is transferred to the gas by collisions between dust particles and gas molecules (see e.g. Gilman 1972; Salpeter 1974; Kwok 1975; Goldreich \& Scoville 1976).

Following these pioneering works, the effect of radiation pressure on dust particles has been studied in more detail by many authors (see e.g. Tielens 1983). So far, however, models used for the interpretation of observed spectra usually assume a prescribed density distribution, typically $\rho \sim r^{-\alpha}$ (see e.g. Justtanont \& Tielens 1992; Danchi et al. 1994; Hashimoto 1994, 1995; Groenewegen 1995), ignoring the fact that the problem of momentum transfer (hydrodynamics) is inherently coupled with the problem of radiative transfer: Radiation pressure on dust determines the outflow velocity and hence the density structure; at the same time, the density structure determines via radiative transfer effects - the spectrum of the photons and hence the effective radiation pressure. Over the last years, this circular problem has been addressed by Netzer \& Elitzur (1993), Habing et al. (1994) and Ivezić \& Elitzur (1995), and it has been found that several different factors influence the efficiency of momentum transfer to the gas and that it is important to describe the gas/dust shell as at least a two fluid system allowing for a relative motion of gas and dust.

For simplicity, however, all of these papers consider only steady state solutions, although it is well known that the stellar parameters and the mass loss rate can undergo significant variations on rather short time scales when so called "thermal pulses" occur on the upper AGB.

We have developed a new code which is suitable to treat time-dependent radiative hydrodynamics, and we have performed calculations similar to those presented by Netzer \& Elitzur (1993), with the aim of first testing our code in the simpler case of constant mass loss rate before applying it to time-dependent calculations of dusty AGB shells. These will take into account the evolutionary changes of the stellar parameters and the mass loss rate of the star at the center of the circumstellar shell over a time interval of several 100000 years, covering the final stages of evolution on the AGB, including several so called "thermal pulses" (with typical interpulse time scales of some $10000 \mathrm{yrs}$ ) but ignoring the dynamical effects of the Mira-type stellar pulsations (with typical periods of some 100 days). For first examples of such time-dependent calculations see Schönberner et al. (1997a,b) and Steffen et al. (1997). One additional goal of this work was to investigate the contribution of the gas pressure to driving the outflow, an effect usually neglected when modeling AGB winds. We have studied the influence of this factor on the outflow velocity and on the efficiency factor $\beta$, the ratio of momentum flux in the wind to momentum flux of the stellar photons.

In Sect. 2 we describe all the equations and assumptions incorporated into our code as well as some simple calculations performed for gray dust opacity in order to check the numerical solution against the analytical one. The main results of our computations for a variety of stellar parameters are presented in Sect. 3, analyzing in detail the properties of the gas and dust outflow velocity and the corresponding spectral energy distributions as a function of mass loss rate. In Sect. 4 we perform an extensive comparison of our results with those presented by Netzer \& Elitzur (1993) and by Habing et al. (1994). Finally, the conclusions are drawn in Sect. 5 .

\section{Hydrodynamical model calculations}

Our model calculations are based on the following picture. The central AGB star has a given mass $\left(M_{*}\right)$, luminosity $\left(L_{*}\right)$, and effective temperature $\left(T_{\text {eff }}\right)$ and loses mass with a prescribed constant mass loss rate $(\dot{M})$. At some distance from the star the outflowing gas has cooled to the dust condensation temperature $\left(T_{\text {cond }}\right)$ and dust is assumed to form instantly at this radius $\left(r_{1}\right)$, which lies very close to the sonic point (critical point). The dust grains of single size are accelerated away from the central star by radiation pressure, drifting through the gas and dragging along the gas component due to the frictional coupling provided by dust-gas collisions.

A 1D radiation hydrodynamics code, originally developed to investigate the dynamical evolution of protostellar clouds, is employed to solve the time-dependent equations of hydrodynamics and frequency-dependent radiative transfer for a two component "fluid" consisting of gas and dust under the assumption of spherical symmetry. Details about the numerical methods used are described by Yorke \& Krügel (1977), and by Yorke (1980a,b). The code has been modified extensively to adapt it for the modeling of dusty stellar outflows. The basic equations to be solved in this context are given below.

\subsection{Hydrodynamics}

The structure of the stellar outflow is governed by the following basic equations of hydrodynamics. 
1) Equation of motion for the gas component:

$$
\begin{aligned}
\frac{\partial u}{\partial t}+u \frac{\partial u}{\partial r} & =-\frac{1}{\rho} \frac{\partial p}{\partial r}-\frac{G M_{\mathrm{r}}}{r^{2}} \\
& +n_{\mathrm{d}} \pi a^{2}(w-u) \sqrt{\bar{v}^{2}+(w-u)^{2}}
\end{aligned}
$$

where $u$ is the gas velocity, $w$ the dust velocity, $\rho$ is the gas density, $p$ is the gas pressure, $M_{\mathrm{r}}$ is the total mass inside radial coordinate $r$, and

$\bar{v}=\sqrt{\frac{8 R T}{\pi \mu}}$

measures the thermal velocity dispersion of the gas molecules at gas temperature $T$ (for the molecular weight we assumed $\mu \approx 1.3$, corresponding to gas of solar composition with hydrogen in atomic form). All other symbols have their usual meaning. The first term on the right hand side of Eq. (1) describes the acceleration due to the gas pressure gradient (gas pressure term), the second term corresponds to the gravitational force and the third term accounts for the dynamical coupling between gas and dust (friction term).

2) Equation of continuity for the gas component:

$\frac{\partial \rho}{\partial t}+\frac{1}{r^{2}} \frac{\partial}{\partial r}\left(r^{2} \rho u\right)=0$

3) Equation of motion for the dust component (single grain):

$$
\begin{aligned}
\frac{\partial w}{\partial t}+w \frac{\partial w}{\partial r} & =\frac{\bar{C}_{\mathrm{ext}}^{F} L_{*}}{m_{\mathrm{d}} 4 \pi r^{2} c}-\frac{G M_{\mathrm{r}}}{r^{2}} \\
& -\frac{\rho}{m_{\mathrm{d}}} \pi a^{2}(w-u) \sqrt{\bar{v}^{2}+(w-u)^{2}}
\end{aligned}
$$

where $m_{\mathrm{d}}$ and $a$ are the mass and radius of a dust grain, respectively, $c$ is the speed of light, and

$\bar{C}_{\text {ext }}^{F}=\int_{0}^{\infty} C_{\text {ext }}(\nu) F(\nu) \mathrm{d} \nu / \int_{0}^{\infty} F(\nu) \mathrm{d} \nu$

is the flux-weighted extinction cross section (including absorption and isotropic scattering: $C_{\text {ext }}(\nu)=C_{\text {abs }}(\nu)+$ $\left.C_{\text {sca }}(\nu)\right)$. The first term on the right hand side of Eq. (4) describes the acceleration of a dust grain by radiation pressure, the second term corresponds to the gravitational deceleration by the central mass (usually much smaller than the radiative acceleration), and the third term again accounts for the dynamical coupling between gas and dust. Interaction between dust particles ("dust pressure") is neglected.

4) Equation of continuity for the dust component:

$\frac{\partial n_{\mathrm{d}}}{\partial t}+\frac{1}{r^{2}} \frac{\partial}{\partial r}\left(r^{2} n_{\mathrm{d}} w\right)=0$ where $n_{\mathrm{d}}$ is the number density of dust grains. Hence, the total amount of dust in the computational domain changes only due to the fluxes through the model boundaries. Condensation or evaporation of dust is not considered.

\subsection{Radiative transfer}

The thermal structure of the dust shell is determined by the radiation field of the central star, which is assumed to radiate as a blackbody with $T_{\mathrm{BB}}=T_{\text {eff }}$ in this work. The energy equation stipulates the condition of radiative equilibrium at any time.

\section{5) Energy equation:}

$\frac{1}{r^{2}} \frac{\partial}{\partial r}\left(r^{2} F_{\text {rad }}\right)=0$

where $F_{\text {rad }}$ is the frequency-integrated radiative energy flux. The temperature of the dust component is related to the equilibrium radiation field by

$$
\int_{0}^{\infty} C_{\text {abs }}(\nu) J_{\nu} \mathrm{d} \nu=\int_{0}^{\infty} C_{\text {abs }}(\nu) B_{\nu}\left(T_{\mathrm{d}}\right) \mathrm{d} \nu
$$

where $J_{\nu}$ is the angle-averaged specific intensity and $B_{\nu}$ is the Planck function at the local dust temperature $T_{\mathrm{d}}$. We presently do not solve the energy equation for the gas component. Instead, we simply take

$T=T_{\mathrm{d}}$

an assumption which needs to be modified for applications where the gas temperature is a critical quantity, like for the calculation of molecular emission line profiles (see also discussion in Sect. 5). For the computation of the radiative transfer (including synthetic spectra) we account only for the absorption, scattering, and thermal emission of the dust grains, whereas the gas component is presently ignored since it is practically transparent in the continuum. The method used for solving the equation of radiative transfer in spherical geometry is described in detail by Yorke (1980b). Briefly, a ray tracing algorithm is used to obtain the frequency-dependent equilibrium radiation field which is usually found after a few iterations. This routine is basically used to find the variable Eddington factors, $f$, which are further used to solve the frequency-independent moment equation for the frequency-integrated mean intensity $J$, taking into account the constraint given by Eq. (7). Solving the moment equation (with given Eddington factors) is much faster than solving the detailed radiative transfer using the ray system. Since the Eddington factors are changing rather slowly with time, ray tracing needs to be repeated only after several hydrodynamical time steps (typically 10) to recompute them. 
The treatment of radiative transfer has been modified according to our needs. In particular, the central star and the dust free region between the stellar surface and the inner boundary of the dust shell is now covered with a better spatial resolution by increasing the number of rays in the central region. We emphasize that this method does not involve any approximations but rather allows a rigorous solution of the radiative transfer problem.

\subsection{Numerical solution}

For the present study we employ time-dependent hydrodynamics to find steady state solutions, starting from a given initial configuration subject to appropriate (timeindependent) boundary conditions. The time needed to achieve steady state is given by the thickness of the shell divided by the mean outflow velocity. Though time consuming, this approach has the advantage of yielding only those solutions which are dynamically stable, at least in $1 \mathrm{D}$.

\subsubsection{Input data}

To pose the problem, we first have to specify the basic properties of the central star which is assumed to radiate as a black body. These are its mass, $M_{*}$, its luminosity, $L_{*}$, its effective temperature, $T_{\text {eff }}$ (which together with $L_{*}$ defines the stellar radius), and the mass loss rate, $\dot{M}$. These quantities are assumed to be constant with time (i.e. we tacitly assume a mass source inside the star to keep $M_{*}$ from changing in accordance with $\dot{M}$ ).

Second, we have to define the properties of the dust grains and their abundances. In this study we use astronomical silicates, graphite or amorphous carbon, with properties as specified in Table 1. The corresponding opacity data for astronomical silicates and graphite were kindly provided by B. Draine (for details see Laor \& Draine 1993), and were taken from Rouleau \& Martin (1991) for amorphous carbon. The wavelength dependence of the absorption and scattering efficiencies,

$Q_{\mathrm{abs}}(\lambda)=\frac{C_{\mathrm{abs}}(\lambda)}{\pi a^{2}} \quad$ and $\quad Q_{\mathrm{sca}}(\lambda)=\frac{C_{\mathrm{sca}}(\lambda)}{\pi a^{2}}$

are displayed in Fig. 1 for the three types of dust, assuming spherical grains with a radius of $a=0.05 \mu \mathrm{m}$. In the hydrodynamical calculations we use a grid of 169 wavelength points distributed between 0.01 and $3100 \mu \mathrm{m}$.

\subsubsection{Boundary conditions}

The distance of the inner edge of the dust shell from the central star, $r_{1}$, is determined during the model calculations by an iterative procedure such that the resulting dust temperature at the inner boundary (according to Eq. (8)) corresponds to to the dust condensation temperature, $T_{\text {cond }}$. The computational radial grid starts at $r_{1}$, the

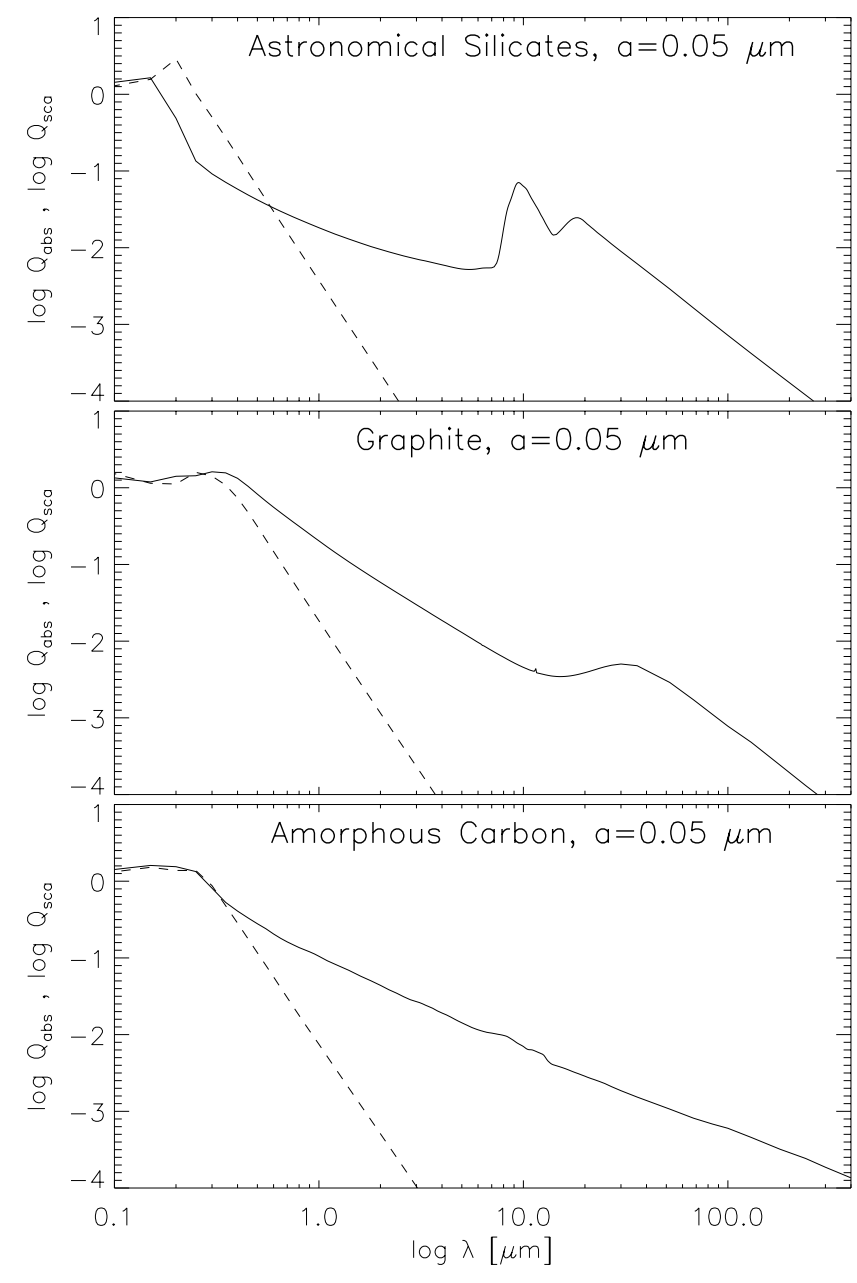

Fig. 1. Wavelength dependence of absorption efficiency $Q_{\text {abs }}$ (solid) and scattering efficiency $Q_{\text {sca }}$ (dashed) for grains of astronomical silicates (top), of graphite (middle) and of amorphous carbon (bottom) adopted in the present work. Note that for the assumed grain size of $a=0.05 \mu \mathrm{m}$, scattering opacity becomes negligible at far infrared wavelengths. For astronomical silicates and for graphite, $Q_{\mathrm{abs}} \sim \lambda^{-2}$ beyond $\lambda \approx 50 \mu \mathrm{m}$. In contrast, the absorption efficiency of amorphous carbon may be roughly approximated by $Q_{\mathrm{abs}} \sim \lambda^{-1}$ over the whole range of infrared wavelengths

inner edge of the dust shell, and ends at an outer radius $r_{\text {out }}=10^{17} \mathrm{~cm}$.

The boundary conditions at $r_{1}$ determine the nature of the solution. At the inner boundary we adopt a constant initial velocity which is identical for the gas and the dust component and is taken to be 2 to $4 \mathrm{~km} / \mathrm{s}$, close to (but not smaller than) the local isothermal sound speed:

$u_{1} \equiv u\left(r_{1}\right)=w\left(r_{1}\right) \approx c_{\mathrm{s}}\left(T_{\text {cond }}\right)$.

The gas density at the inner boundary is then given by

$\rho\left(r_{1}\right)=\frac{\dot{M}}{4 \pi r_{1}^{2} u_{1}}$ 
the dust density is related to the gas density via the dustto gas ratio $\delta$ as

$\rho_{\mathrm{d}}\left(r_{1}\right)=\delta \rho\left(r_{1}\right)$.

At the outer boundary, $u, w, \rho$ and $\rho_{\mathrm{d}}$ can be computed consistently from the hydrodynamical equations in the case of a supersonic outflow considered here. Since no information can travel into the computational domain from outside, there is no necessity (and no possibility) to pose boundary conditions for the hydrodynamics at the outermost grid point. But we need to specify a boundary condition for the solution of the radiative transfer. We assume that the "interstellar" radiation field incident on the outer boundary from outside is that of a blackbody at $T_{\mathrm{BB}}=3 \mathrm{~K}$.

\subsubsection{Test calculations}

In the case of gray opacity, $\bar{C}_{\text {ext }}^{F}$ becomes independent of the radiation field and hence is no longer a function of of $r$. Assuming, in addition, a very strong coupling between gas and dust such that the drift velocity $w-u$ becomes negligible, and further ignoring gas pressure, the equation of motion can be solved analytically. It is worth noting that the well known solution for $v \equiv u=w$,

$v^{2}(r)=v^{2}\left(r_{1}\right)+\left(\frac{\chi_{\mathrm{F}} L_{*}}{2 \pi c r_{1}}-\frac{2 G M_{*}}{r_{1}}\right)\left(1-\frac{r_{1}}{r}\right)$,

is independent of the mass loss rate. The flux-averaged opacity per unit mass, $\chi_{\mathrm{F}}$, is related to $\bar{C}_{\mathrm{ext}}^{F}$ via

$\chi_{\mathrm{F}}=\frac{\delta \bar{C}_{\mathrm{ext}}^{F}}{m_{\mathrm{d}}} \frac{u}{w}$.

Introducing the ratio between radiative to gravitational acceleration,

$\Gamma=\frac{\chi_{\mathrm{F}} L_{*}}{4 \pi c G M_{*}}$,

(which is a constant in the gray case), Eq. (14) may be rewritten as

$$
\begin{aligned}
v^{2}(r) & =v^{2}\left(r_{1}\right)+\frac{2 G M_{*}}{r_{1}}(\Gamma-1)\left(1-\frac{r_{1}}{r}\right) \\
& =v^{2}\left(r_{1}\right)+v_{\mathrm{esc}}^{2}\left(r_{1}\right)(\Gamma-1)\left(1-\frac{r_{1}}{r}\right)
\end{aligned}
$$

where

$v_{\mathrm{esc}}\left(r_{1}\right)=\sqrt{\frac{2 G M_{*}}{r_{1}}}$

is the escape velocity at the dust formation radius $r_{1}$. We have checked that our numerical code finds the correct solution for this test case of gray dust opacity and vanishing drift velocity. As expected, numerical errors of the order $\Delta r / r$ (where $\Delta r$ is the numerical resolution of the radial grid) are found for the resulting velocity relative to the analytical solution, the numerical velocities being systematically smaller. A proper choice of the radial grid ensures that these kinds of errors are kept below $\approx 1 \%$.

All models used for this investigation have $N=240$ radial grid point spaced according to

$r_{i}=r_{1}+\left(r_{N}-r_{1}\right) \frac{q^{i-1}-1}{q^{N-1}-1} ; i=1, N$.

By choosing an appropriate value $q>1$ we have concentrated the grid points in the inner part of the model such that the spatial resolution in the acceleration region is four times better than for an equidistant logarithmic grid covering the same radius range with the same number of points. The resulting lower resolution in the outer parts is not critical since here all quantities approach their asymptotic limits and are almost constant with $r$.

Table 1. Dust grain properties and abundances used in this work. The corresponding optical properties are shown in Fig. 1

\begin{tabular}{|l|c|c|c|}
\hline & $\begin{array}{c}\text { Astronomical } \\
\text { Silicates }\end{array}$ & Graphite & $\begin{array}{c}\text { Amorphous } \\
\text { Carbon }\end{array}$ \\
\hline \hline Grain size $[\mu \mathrm{m}]$ & 0.05 & 0.05 & 0.05 \\
\hline $\begin{array}{l}\text { Specific mass density } \\
\rho_{\mathrm{i} \quad\left[\mathrm{g} \mathrm{cm}^{-3}\right]}\end{array}$ & 3.30 & 2.26 & 1.85 \\
\hline $\begin{array}{l}\text { Condensation } \\
\text { temperature }[\mathrm{K}]\end{array}$ & 800 & 850 & 850 \\
\hline \hline $\begin{array}{l}\text { Dust-to-gas ratio } \\
\delta=\rho_{\mathrm{d}} / \rho\end{array}$ & 0.0050 & 0.0015 & 0.0015 \\
\hline \hline
\end{tabular}

\section{A sample of steady state models}

In the following we present the resulting steady state solutions for different stellar parameters and mass loss rates. Three sets of models are considered, the first assuming oxygen-rich chemistry with silicate dust (Figs. 2 to 5 , Tables 2 to 5 ), the other two being based on carbonrich chemistry with graphite dust (Figs. 6 to 8, Tables 6 to 8 ) and grains of amorphous carbon (Figs. 9 to 11, Tables 9 to 11), respectively. The figures show the resulting steady state velocity field (gas and dust component) and the corresponding combined spectral energy distribution of the star and the dusty outflow for a representative sample of models. Details about the stellar parameters used and other input data are given in the figure captions. Additional information can be found in the tables which list the basic results for all the steady state models investigated here. For each set of input parameters, two runs were carried out, one including the effects of gas pressure in the equations of motion, the other assuming no gas pressure. Note that for some examples, the adopted mass loss rates are so high that a significant fraction of the stellar mass is expelled during the flight time of a gas parcel 
through the wind shell. In these cases, steady state models are certainly not adequate.

\subsection{Velocity structure}

From the analysis of the present sample of steady state models we can draw the following conclusions about the properties of dust-driven winds around late type stars:

a) The acceleration of the dust and gas takes place in a narrow region close to the dust formation point $\left(r_{1}<\right.$ $\left.r<r_{2} \approx 5 r_{1}\right)$. Although in general $\Gamma$ is not constant but depends on $r$, this is in qualitative agreement with Eq. (17) which suggests that $90 \%$ of the terminal velocity is reached at $r \approx 5 r_{1}$.

b) For low mass loss rates, the drift velocity of the dust relative to the gas, $v_{\text {drift }}=w-u$, may amount to several times the gas velocity and reduces the effective dust opacity (per unit volume) by a factor $u / w$. In general, $w \approx 2 u$ (used by e.g. Le Sidaner \& Le Bertre 1996) is a reasonable approximation only for low to moderate mass loss rates, while $w \approx u$ becomes valid for very high mass loss rates.

c) For high mass loss rates, i.e. large (flux-averaged) optical depths, the momentum flux in the outflow, $\dot{M} u_{\mathrm{e}}$ can exceed the momentum flux of the photons, $L_{*} / c$. The ratio of these quantities, $\beta=\dot{M} u_{\mathrm{e}} c / L_{*}$, is listed in Tables 2 to 11 and can be seen to exceed unity in a number of cases. In fact, several stars with $\beta>1$ are known and it is now clear that there is no physical reason preventing the outflow from crossing the $\beta=1$ border (see e.g. Nezter \& Elitzur 1993; Habing et al. 1994; or Ivezić \& Elitzur 1995 for a discussion of this issue).

d) As expected, the outflow velocity, $u_{\mathrm{e}}$, depends on stellar mass, luminosity, grain properties and dust abundance. But in contrast to our simple gray model (cf. Eq. 17), $u_{\mathrm{e}}$ depends also on the stellar effective temperature, $T_{\text {eff }}$, and on the mass loss rate. This is because the extinction cross section of realistic dust grains is strongly wavelength dependent (see Fig. 1), and hence the fluxaveraged opacity, $\chi_{\mathrm{F}}$, the quantity determining the net radiative acceleration, depends on the spectral energy distribution of the stellar radiation $\left(T_{\text {eff }}\right)$ and on the degree of reprocessing of the stellar radiation by the dust shell, which increases with the optical depth of the dust shell, i.e. with the mass loss rate.

e) The terminal outflow velocity, $u_{\mathrm{e}}$, is of the order of the escape velocity at the dust formation point, $v_{\mathrm{esc}}\left(r_{1}\right)$. Since

$u_{\mathrm{e}} \approx v_{\mathrm{esc}}\left(r_{1}\right) \sqrt{\Gamma_{\mathrm{eff}}-1}$

(see also Eq. 17) with the effective $\Gamma$ given by

$\Gamma_{\mathrm{eff}}=r_{1} \int_{r_{1}}^{\infty} \frac{\Gamma(r)}{r^{2}} \mathrm{~d} r$ (essentially the average $\Gamma$ in the acceleration region), outflow velocities in the range 1 to 3 times $v_{\text {esc }}\left(r_{1}\right)$ imply effective $\Gamma \mathrm{s}$ in the range 2 to 10 .

f) It has often been argued that the acceleration due to the gas pressure gradient may be neglected because the outflow is highly supersonic (e.g. Habing et al. 1994). However, this condition is not really fulfilled by the relatively slow outflows around AGB stars where the ratio $u_{\mathrm{e}} / c_{\mathrm{S}}\left(r_{1}\right)$ hardly exceeds 10 .

The gas pressure term $\frac{1}{\rho} \frac{\partial p}{\partial r}$ can safely be neglected if it is much smaller than the advection term $u \frac{\partial u}{\partial r}$ over most of the acceleration region. For the wind shells investigated here, the ratio of pressure term to advection term can be evaluated analytically assuming a velocity structure according to Eq. (17) with a gas temperature falling like $r^{-1 / 2}$. The result is

$Y \equiv\left(\frac{1}{\rho} \frac{\partial p}{\partial r}\right) /\left(u \frac{\partial u}{\partial r}\right)=5 \sqrt{x} \frac{\epsilon^{2}}{1-\epsilon^{2}}+\frac{\epsilon^{2} \sqrt{x}}{x-\left(1-\epsilon^{2}\right)}$,

where $x=r / r_{1}$ is the dimensionless radial coordinate, and $\epsilon=u_{1} / u_{\mathrm{e}} \approx c_{\mathrm{S}}\left(r_{1}\right) / u_{\mathrm{e}}$ is the ratio of initial to final gas velocity, which approximately equals the inverse ratio of outflow velocity to sound speed at the inner radius of the shell. Near $x=1, Y$ is close to 1 since the flow starts with $u \approx c_{\mathrm{s}}$ at the dust formation point $r_{1}$. For small $\epsilon<0.05$, $Y$ decreases sharply as $x$ increases and becomes negligible for $x>1.2$, so the overall effect of the gas pressure term is small. However, the situation is different for larger $\epsilon>0.05$ where $Y$ attains a minimum near $x=1.7$ and increases again for larger $x$. In the case $\epsilon=0.2$, for example, $Y$ is larger than 0.35 everywhere, so the gas pressure term is not at all negligible. Indeed, comparison of the runs with and without the effects of gas pressure shows that, in general, the gas pressure term leads to significantly higher outflow velocities (see Figs. 15, 16, 17 and tables). Only if the radiative acceleration is strong enough to produce highly supersonic outflows $\left(u_{\mathrm{e}}>30 \mathrm{~km} / \mathrm{s}\right)$ the gas pressure term becomes unimportant. This is what we see in our models with the highest luminosities ("supergiant" models $\mathrm{E}$ and F, see Tables 4 and 5 as well as Fig. 15).

g) For decreasing mass loss rate, the coupling between dust and gas diminishes and the drift velocity increases monotonically. Consequently, the effective $\Gamma$ decreases because of the factor $u / w$ (cf. Eqs. (15), (16)). The minimum mass loss rate is reached for $\Gamma_{\text {eff }}=1$. No dust driven wind is possible below this critical mass loss rate. We have refrained from calculating these minimum mass loss rates from our models because we found it to be very sensitive to the assumed gas pressure (see models C6_m and C6_n, Table 2 or models J6_m and J6_n, Table 9). Lower mass loss rates can be sustained when gas pressure supports the outflow. In addition, it must be kept in mind that some kind of shock wave pressure is generated by the stellar pulsation which will reduce the minimum mass loss rate even further. 
h) It has been noted above that the outlow velocity is independent of $\dot{M}$ for gray dust (cf. Eq. 17), so there is no maximum possible mass loss rate. However, the situation is different for realistic dust opacities which strongly decrease with wavelength (cf. Fig. 1). As the mass loss rate is increased the optical depth of the dusty envelope is increased as well, and the maximum of the spectral energy distribution of the radiation, as seen by a dust particle in the acceleration region of the shell, is shifted to longer wavelengths. As a consequence, the flux averaged extinction cross section (cf. Eq. 5) is decreased and the effective $\Gamma$ is reduced (cf. Eqs. (15), (16)). This mechanism puts an upper limit to the mass loss rate, the quantitative value of which depends on the stellar parameters. Again, we find this limit to be sensitive the the assumptions about the gas pressure. For example, model I1 can drive a mass loss of $210^{-4} M_{\odot} /$ yr only if supported by gas pressure (see Table 8).

i) Test calculations have confirmed that the assumption of complete momentum coupling is an excellent approximation. Under this assumption, the drift velocity, $v_{\text {drift }}=w-u$ is given by (see e.g. Tielens 1983)

$$
\begin{aligned}
& v_{\mathrm{drift}}^{2}= \\
& \frac{1}{2}\left\{\sqrt{\left[\left(\frac{4 u}{a^{2} \dot{M}}\right)^{2}\left(\frac{\bar{C}_{\mathrm{ext}}^{F} L_{*}}{2 \pi c}-2 G M_{\mathrm{r}} m_{\mathrm{d}}\right)^{2}+\bar{v}^{4}\right]}-\bar{v}^{2}\right\} .
\end{aligned}
$$

j) We note that for the parameters used in this study, the resulting gas outflow velocities are in the range 5 to $40 \mathrm{~km} / \mathrm{s}$, covering the range of observed outflow velocities.

\subsection{Spectral energy distributions}

Along with the velocity structure, Figs. 2 to 11 show the corresponding stellar input spectrum (dot-dashed) and the emergent spectral energy distribution (SED) of the star plus circumstellar dust shell (solid line). From the analysis of this sample of spectra we infer the following properties.

a) The shape of the SEDs changes monotonically with mass loss rate, i.e. with the optical depth of the dust shell. Oxygen stars with low mas loss rates show the characteristic silicate features $(\lambda 9.7$ and $18 \mu \mathrm{m})$ in emission, while the overall shape of the SED closely corresponds to that of the central star. For intermediate mass loss rates, the SED is shifted to the red through processing of photons by the dust shell, and self absorption begins to become important, noticeable by a central absorption in the main silicate feature which is, however, still seen in emission (see model D3_m, center of Fig. 3). For even higher mass loss rates, the stellar SED is no longer visible (IR objects) and the silicate features turn into strong absorption.

b) Analogous changes occur for Carbon stars, although the lack of features in the opacity of carbon-based grains makes it more difficult to detect subtle differences in the spectra of models with slightly different mass loss rates. c) For given dust properties, the shape of the SED is determined, to a first approximation, only by the total optical depth of the dust shell (at some reference wavelength), and is essentially independent of the stellar luminosity (see also Ivezić \& Elitzur 1995).

d) In principle, the sequences of models presented here can be used to estimate the mass loss rates of Oxygen and Carbon AGB stars by finding the model which most closely matches the observed SED together with the observed (gas) outflow velocity. The real mass loss rate may differ from that of the model identified in this way if the star to be analyzed has a different luminosity or dust-togas ratio than assumed in the model. However, it can be shown that in this case the scaling relation

$\dot{M} \delta / \sqrt{L_{*}}=$ const.

can be used to derive the actual mass loss rate if $L_{*}$ and $\delta$ are known independently (see e.g. Eq. (1) in Groenewegen 1995).

e) To provide a reference for comparison with the time-dependent calculations to be presented in a forthcoming paper (for first results see Schönberner et al. 1997a,b; Steffen et al. 1997), we have calculated the positions of all of our steady state models in two different IRAS two-color-diagrams $\left(F_{\nu}(60 \mu \mathrm{m}) / F_{\nu}(25 \mu \mathrm{m})\right.$ versus $F_{\nu}(25 \mu \mathrm{m}) / F_{\nu}(12 \mu \mathrm{m})$ and $F_{\nu}(100 \mu \mathrm{m}) / F_{\nu}(60 \mu \mathrm{m})$ versus $F_{\nu}(25 \mu \mathrm{m}) / F_{\nu}(12 \mu \mathrm{m})$, see Figs. 12 to 14$)$. The IRAS colors were computed by convolution of the emergent synthetic spectrum with the IRAS band profiles, taking into account the wavelength dependence of the spectra assumed by the IRAS team (i.e. $F_{\lambda} \sim \lambda^{-1}$, see the IRAS Explanatory Supplement 1985). The models with the lowest mass loss rates are found to the left of these diagrams, while the models with the highest mass loss rates are located at far right. We note that for fixed dust properties, all models fall on a simple color-color relation with $\dot{M}$ (or optical depth) as the only parameter, in agreement with the results presented by Ivezic \& Elitzur (1995, see their Fig. 6). These diagrams also demonstrate the effect of the gas pressure on the computed IRAS colors, which is clearly noticeable for most of the AGB stars while it is negligible for the supergiants.

f) For a number of cases we have computed "simple" comparison models in order to illustrate the difference between synthetic spectra computed from these models and those resulting from our self-consistent hydrodynamical approach. The simple models are constructed by assuming a constant outflow velocity equal to the terminal gas outflow velocity of the respective hydrodynamical model (i.e. $\rho \sim r^{-2}$ ), and ignoring dust drift (i.e. $u \equiv w$ ). The comparison of the SEDs is shown in Figs. 3, 6, and 9. We were surprised to see the relatively close agreement between both sets of models. Obviously, the effects of assuming a constant outflow velocity and neglecting the dust drift velocity cancel to some degree. We conclude that "simple" models may be used for the analysis of observed SEDs 
without introducing large systematic errors, provided the adopted constant outflow velocity equals the observed one.

\section{Comparison with the results of different authors}

The input parameters used for the calculation of the steady state models presented in the previous section were chosen such that they match exactly those used by Netzer \& Elitzur (1993, hereafter NE) who list the results of similar dust-driven wind calculations for a variety of parameters in their Table 3 (missing information about input data, e.g. $T_{\text {cond }}, u_{1}$, were kindly communicated to us upon request by these authors). When comparing our results with those of NE one has to be aware that NE ignored the gas pressure term $\frac{1}{\rho} \frac{\partial p}{\partial r}$ in Eq. (1) and the thermal velocity dispersion of the gas molecules, $\bar{v}$, in the friction term (cf. Eqs.(1), (2) and (4)), which is equivalent to assuming zero temperature for the gas. For the purpose of a direct comparison, we changed our code such that the above mentioned terms can be ignored and recomputed all cases for vanishing gas pressure and $\bar{v}$ (model names ending with _n). The results of these calculations should be directly comparable to the data given in NE's Table. 3.

The comparison for different Oxygen stars with dust composed of astronomical silicates in their circumstellar shells (cf. panels $\mathrm{C}$ to F of NE's Table 3 ), each with a sequence of mass loss rates, is presented in Fig. 15. We notice that neither the shape of the relation $u_{\mathrm{e}}(\dot{M})$ nor the values of the terminal gas velocities $u_{\mathrm{e}}$ (except for the lowest mass loss rates) are in reasonable agreement. In particular, all of our models show a maximum outflow velocity at intermediate mass loss rates and a distinct decrease of $u_{\mathrm{e}}$ towards higher mass loss rates, while the NE models exhibit monotonic increase of $u_{\mathrm{e}}$ with mass loss rate. Moreover, note that we were not able to find a steady state solution for the lowest mass loss rate in panel $\mathrm{C}$ without the support of gas pressure. In this case we found the gas to fall inwards; the coupling between dust and gas was not sufficient to drive the wind.

A similar comparison for Carbon stars with graphite dust in their circumstellar shells (cf. panels G to I of NE's Table 3 ) is shown in Fig. 16. Here the shape of our relation $u_{\mathrm{e}}(\dot{M})$ is in qualitative agreement with the results of NE. However, in all cases our terminal gas velocities computed without gas pressure are significantly lower than those given by NE. For the highest mass loss rate in panel I we were not able to find a steady state solution without the support of gas pressure.

To find out the reason for these differences, a number of models from NE's Table 3 were recomputed by Z. Ivezić (private communication), using exactly the same opacities as used in the present work, and using a code similar to that of NE, but with an improved version of the radiative transfer. The results found from these test calculations are very similar to ours.
For completeness and future reference, we have recomputed all the Carbon star models assuming the dust grains to be composed of amorphous carbon instead of graphite (such models were not considered by NE). The results are displayed in Figs. 9 to 11 and are listed in more detail in Tables 9 to 11 . The terminal outflow velocities, $u_{\mathrm{e}}$, as a function of mass loss rate are shown in Fig. 17. They are qualitatively very similar to those obtained with graphite dust (cf. Fig. 16). Quantitatively, the velocities are somewhat higher, especially for high mass loss rates. For models $\mathrm{J}$, we have computed additional models assuming a more realistic molecular weight of $\mu=2.33$ (molecular hydrogen) instead of the standard assumption $\mu \approx 1.3$ (atomic hydrogen). As expected, the results are between those for $\mu \approx 1.3$ and those obtained ignoring the gas pressure terms $(\mu=\infty)$. We note that also for $\mu=2.33$ the gas pressure leads to a noticeable increase of the expansion velocity.

A similarly detailed comparison with the work by Habing et al. (1994) (hereafter HTT) turns out to be impossible due to the lack of information about a variety of input parameters used in their computations. So we have to restrict our comparison to a few qualitative remarks.

In their Fig. 5, HTT show the variation of the gas velocity as a function of the prescribed mass loss rate for an Oxygen star model with $L_{*}=10^{4} L_{\odot}$ and $\delta=410^{-3}$. These models are roughly comparable with our models $\mathrm{C}\left(L_{*}=10^{4} L_{\odot}\right.$ and $\left.\delta=510^{-3}\right)$. The velocities obtained by HTT are quite similar to ours (considering only models computed without gas pressure). Existing differences may be related to different assumptions about the stellar mass, the dust condensation temperature, and the dust opacity which, according to Fig. 10 of HTT, is about a factor of two higher than ours (different grain size?). Nevertheless, the qualitative agreement with our results is much better than in the case of NE. In contrast to NE, both the results of the present work and those of HTT indicate that the outflow velocity attains a maximum near $\dot{M}=10^{-5} M_{\odot} /$ yr and decreases again towards higher mass loss rates.

We have also tried to reproduce the $\beta\left(\tau_{10}\right)$ relations plotted in Figs. 6 and 7 of HTT. Our results, shown in the left-hand column of Fig. 18, demonstrate that there is no unique $\beta\left(\tau_{10}\right)$ relation neither for Oxygen nor for Carbon stars, in contrast to what is suggested by the work of HTT. Rather, $\beta\left(\tau_{10}\right)$ depends strongly on the stellar parameters. Compare, for example, the relations for models $\mathrm{K}$ and $\mathrm{L}$ (bottom panel), which differ only in the adopted stellar mass. Similarly, the relations for models D and E (top panel) are significantly different, indicating that $\beta\left(\tau_{10}\right)$ depends sensitively on the stellar luminosity. The relations are expected to depend also on the adopted dust-to-gas ratio and on the grain properties.

HTT's $\beta\left(\tau_{10}\right)$ relation for Oxygen stars is much steeper than the steepest one found in our set of models (ignoring gas pressure). It seems impossible to pin down the reason for this discrepancy since there is no precise information 
about the parameters used by HTT to produce the results shown in their figure. Similar remarks apply to the comparison for Carbon stars with amorphous carbon dust.

In Fig. 18 we also present the corresponding $\beta\left(\tau_{\mathrm{F}}\right)$ relations (right-hand column), where $\tau_{\mathrm{F}}$ the flux-averaged optical depth defined as

$\tau_{\mathrm{F}}=\int_{r_{1}}^{\infty} \chi_{\mathrm{F}} \rho(r) \mathrm{d} r$.

It can be shown that, at least in the case of no dust drift and no gas pressure,

$\beta=\tau_{\mathrm{F}}\left(1-\bar{\Gamma}^{-1}\right)$,

(for details see Ivezić \& Elitzur, Eq. (5)). We note that the $\beta\left(\tau_{\mathrm{F}}\right)$ relations shown in Fig. 18 can rarely be approximated by a linear relationship and that their slope depends on the stellar parameters just like in the case of $\beta\left(\tau_{10}\right)$. The reason for this variation is that for the AGB stars considered here, $\bar{\Gamma}$ is not much larger than unity (cf. Eq. 26). Only the supergiants (models E) realize the limiting case $\beta=\tau_{\mathrm{F}}$.

Of course the $\beta(\tau)$ relations become significantly steeper when the flow is supported by gas pressure. This is also demonstrated in Fig. 18, where for models $\mathrm{D}, \mathrm{H}$ and $\mathrm{K}$ the $\beta(\tau)$ relations computed with gas pressure terms are plotted for comparison.

In summary, we conclude that neither the $\beta\left(\tau_{10}\right)$ nor the $\beta\left(\tau_{\mathrm{F}}\right)$ relations are particularly useful for deriving additional information about the physical parameters of dusty stellar outflows from their observable properties.

\section{Concluding remarks}

We have shown that our radiation hydrodynamics code is capable of finding steady state solutions for stellar outflows driven by radiation pressure on dust for a wide variety of stellar parameters and mass loss rates. We have checked that the code finds the correct solution for the case of gray dust opacities and no drift velocity, for which an analytical solution is easily obtained. Numerical errors of the order $\Delta r / r(\Delta r$ : numerical resolution of the radial grid) are kept below $\approx 1 \%$ by a proper choice of the radial grid.

For more realistic cases with non-gray dust opacities and including drift of dust relative to gas, we compared our results with those published by Netzer \& Elitzur (1993). For Oxygen stars we notice that neither the shape of the relation $u_{\mathrm{e}}(\dot{M})$ nor the values of the terminal gas velocities $u_{\mathrm{e}}$ (except for the lowest mass loss rates) are in reasonable agreement, in some cases differing by a factor of 2 . While all of our models show a maximum outflow velocity at intermediate mass loss rates $\left(\dot{M} \approx 10^{-5} M_{\odot} /\right.$ yr $)$ and a distinct decrease of $u_{\mathrm{e}}$ towards higher mass loss rates, the models of $\mathrm{NE}$ exhibit a monotonic increase of $u_{\mathrm{e}}$ with mass loss rate. Although NE give an explanation for this behavior, we believe that our models are more appropriate. Qualitatively, our results for Oxygen stars are confirmed by the work of Habing et al. (1995).

We have tried to identify the reason for this discrepancy with respect to NE. For instance, we have checked that the gravitational force of the shell itself, which is included in our code but is ignored by NE, is no explanation. For a few examples, we have investigated the effect of arbitrarily changing the absorption cross section by $20 \%$ at all wavelengths. We conclude that uncertainties of this magnitude are not sufficient to bring our results into agreement with NE. Moreover, we checked that the opacities for astronomical silicates and graphite computed on the basis of Mie theory from the dielectric constants given by Draine (1987) (used by NE) are in excellent agreement with the opacities used in the present work. Test calculations suggest that certain approximations in the treatment of radiative transfer adopted by NE, in particular the Adams \& Shu (1986) closure relation applied frequency by frequency, may lead to large errors in the case of optically thick dust shells. For some reason, this effect seems to be more pronounced for silicate dust. Another difference is that our radiative transfer scheme resolves the central star while NE treat it as a point source, which may be a questionable approximation.

In addition, we have checked our code against a completely independent radiative transfer code (DUSTCD, cf. Leung 1975, 1976; Egan et al. 1978) suitable for the computation of the infrared radiation field of dusty stellar envelopes. In all cases investigated (optically thin and optically thick winds, gray opacities) we found excellent agreement. Note that in order to achieve a reasonable energy conservation with DUSTCD, a sufficient spatial resolution in the acceleration region of the shell was found to be essential.

Finally we would like to point out that our test calculations have clearly demonstrated that, in general, gas pressure cannot be neglected in the equation of motion. Our results show that in practice gas pressure is important unless the terminal outlow velocities are really highly supersonic, i.e. $u_{\mathrm{e}} / c_{\mathrm{s}}\left(r_{1}\right)>20$. For slow AGB winds $\left(u_{\mathrm{e}} \approx 10 \mathrm{~km} / \mathrm{s}\right)$, the outflow velocities may become up to $50 \%$ larger (cf. Figs. 15 to 17) if gas pressure is allowed to support the wind. These conclusions are based on the assumption that $T=T_{\mathrm{d}}$ and a mean molecular weight of the gas of $\mu \approx 1.3$. Actually, $\mu=2.33$, corresponding to hydrogen in molecular form, may be more appropriate and would reduce the importance of gas pressure roughly by a factor of two. On the other hand, $T$ may actually be higher than $T_{\mathrm{d}}$ due to frictional heating (Krüger et al. 1994), leading to a corresponding enhancement of the role of gas pressure, especially in the case of large drift velocity, i.e. low mass loss rates (Kastner 1992).

Along with the hydrodynamics of AGB winds, we have computed the emergent spectral energy distribution (SED) of the star plus circumstellar dust shell. From the 
analysis of this sample of spectra we infer that, for fixed dust properties, all models fall on a simple color-color relation in the IRAS two-color-diagrams, with $\dot{M}$ (or optical depth) being the only parameter.

Surprisingly, we found a close agreement between the synthetic spectra resulting from the self-consistent hydrodynamical approach and those obtained from much simpler models based on a constant outflow velocity and ignoring dust drift. Obviously, the effects of assuming a constant outflow velocity and neglecting the dust drift velocity cancel to some degree. We conclude that "simple" models may be used for the analysis of observed SEDs without introducing large systematic errors, provided the adopted constant outflow velocity equals the observed one.

This study constitutes the basis for future timedependent hydrodynamical calculations. In a subsequent paper we will investigate the dynamical response of circumstellar gas/dust shells to the temporal variations of the stellar parameters and mass loss rate. To our knowledge, this problem has not yet been addressed by real radiation hydrodynamics calculations, although it is well known that the stellar parameters and the mass loss rate can undergo significant variations on rather short time scales when intermediate mass stars experience so called "thermal pulses" on the upper AGB.

Acknowledgements. This research has been funded by "Deutsche Agentur für Raumfahrtangelegenheiten" (DARA) under grant 50 OR 9411. One of us (R. Sz.) expresses his gratitude to the Canadian Institute of Theoretical Astrophysics and to the Polish State Committee for Scientific Research - grant No. 2.P03D.027.10. A.M. and R.Sz. are grateful for the hospitality and support provided by the Astrophysical Institute Potsdam. We are indebted to H. Yorke for the permission to modify his original code and to apply it to the problem of dusty stellar outflows.

\section{References}

Adams F.C., Shu F.H., 1986, ApJ 308, 836

Danchi W.C., Bester M., Degiacomi C.G., Greenhill L.J.,
Townes C.H., 1994, AJ 107, 1469

Draine B.T., 1987, Princeton University Observatory preprint No. 213

Egan M.P., Leung C.M., Spagna G.F., 1978, Comp. Phys. Comm. 48, 271

Gilman R.C., 1972, ApJ 178, 423

Goldreich P., Scoville N., 1976, ApJ 205, 144

Groenewegen M.A.T., 1995, A\&A 293, 463

Habing H.J., Tignon J., Tielens A.G.G.M., 1994, A\&A 286, 523

Hashimoto O., 1994, A\&AS 107, 445

Hashimoto O., 1995, ApJ 442, 286

IRAS Explanatory Supplement, Beichman C.A., Neugebauer C., Habing H.J., Cleeg P.E., Chester T.J. (eds.), 1985, JPL, Pasadena

Ivezić Z., Elitzur M., 1995, ApJ 445, 415

Justtanont K., Tielens A.G.G.M., 1992, ApJ 389, 400

Kastner J.H., 1992, ApJ 401, 37

Krüger D., Gauger A., Sedlmayr E., 1994, A\&A 290, 573

Kwok S., 1975, ApJ 198, 583

Laor A., Draine B.T., 1993, ApJ 402, 441

Le Sidaner P., Le Bertre T., 1996, A\&A 314, 896

Leung C.M., 1975, ApJ 199, 340

Leung C.M., 1976, ApJ 209, 75

Netzer N., Elitzur M., 1993, ApJ 410, 701

Rouleau F., Martin P.G., 1991, ApJ 337, 526

Salpeter E.E., 1974, ApJ 193, 585

Schönberner D., Steffen M., Stahlberg J., Kifonidis K., Blöcker T., 1997a, in The Carbon Star Phenomenon, Proc. IAU Symp. 177, Wing R.F. (ed). Kluwer Academic Publishers (in press)

Schönberner D., Steffen M., Stahlberg J., Kifonidis K., Blöcker T., 1997b, in Advances in Stellar Evolution, Rood R.T. and Renzini A. (eds.). Cambridge University Press (in press)

Steffen M., Szczerba R., Men'shchikov A., Schönberner D., 1997, in Advances in Stellar Evolution, Rood R.T. and Renzini A. (eds). Cambridge University Press (in press)

Tielens A.G.G.M., 1983, ApJ 271, 702

Yorke H.W., Krügel E., 1977, A\&A 54, 183

Yorke H.W., 1980a, A\&A 85, 215

Yorke H.W., 1980a, A\&A 85, 215

Yorke H.W., 1980b, A\&A 86, 286 
Stellar parameters: $M_{*}=1 M_{\odot}, T_{\text {eff }}=3000 \mathrm{~K}, L_{*}=1 \times 10^{4} ;$ Dust: astronomical silicates, $a=0.05 \mu m, \delta=0.005$
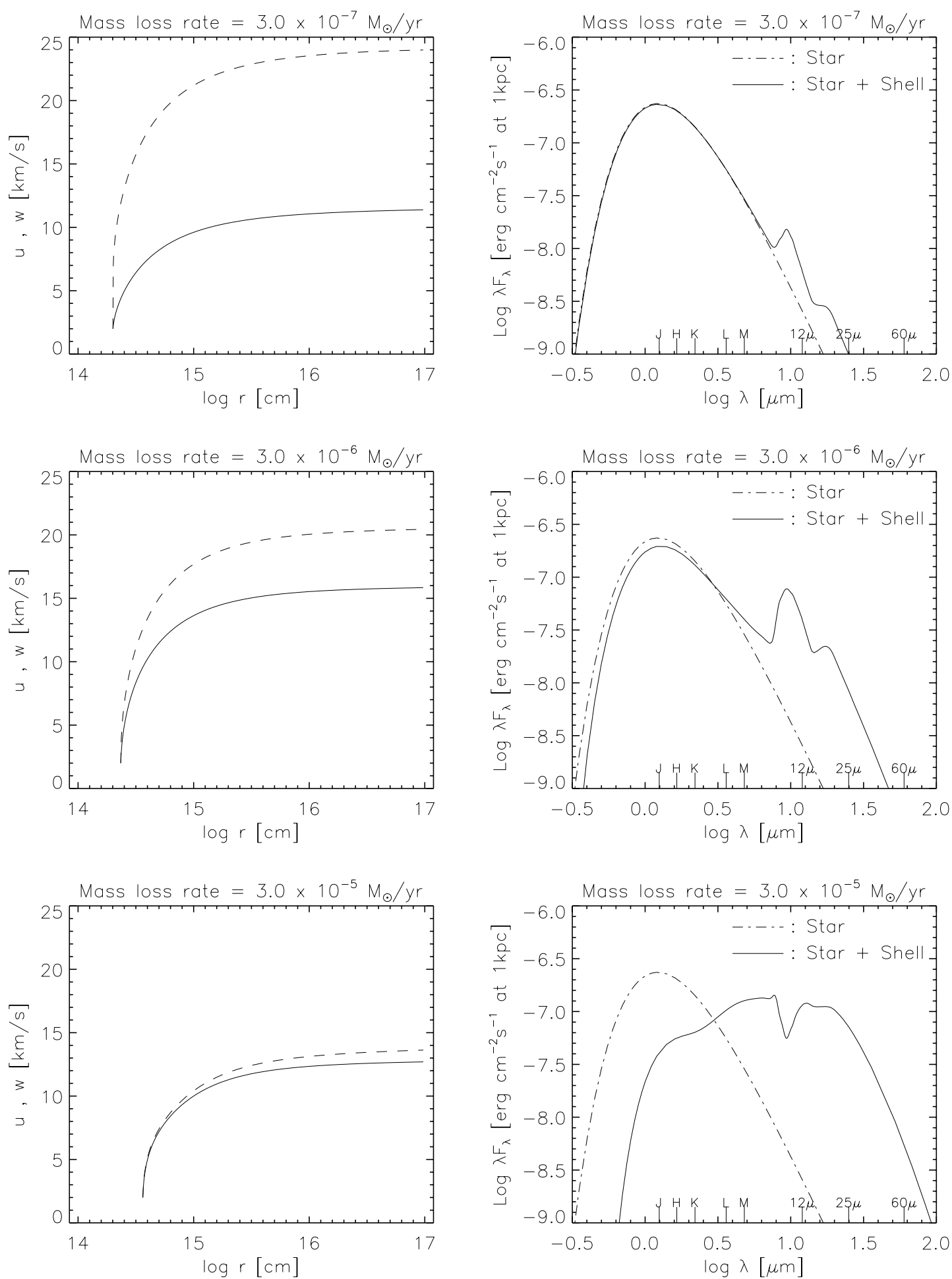

Fig. 2. Model results for an Oxygen star with $M_{*}=1 M_{\odot}, L_{*}=1.010^{4} L_{\odot}$, and $T_{\text {eff }}=3000 \mathrm{~K}$ for 3 different mass loss rates (indicated in the title of each panel). The dust is assumed to consist of grains of astronomical silicates with properties given in Table 1. The initial velocity at the dust formation point is assumed to be $u_{1}=2 \mathrm{~km} / \mathrm{s}$. The left hand panels show the gas velocity (solid) and the dust velocity (dashed) as a function of radial distance. The right hand panels show the corresponding stellar input spectrum (dot-dashed) and the emergent spectral energy distribution (solid) which is the result of processing of the stellar radiation by the dusty envelope. More information about these models (C6_m, C3_m, C1_m) and additional runs of this sequence is listed in Table 2 
Stellar parameters: $M_{*}=4 M_{\odot}, T_{\text {eff }}=2500 \mathrm{~K}, L_{*}=5 \times 10^{4}$; Dust: astronomical silicates, $a=0.05 \mu m, \delta=0.005$
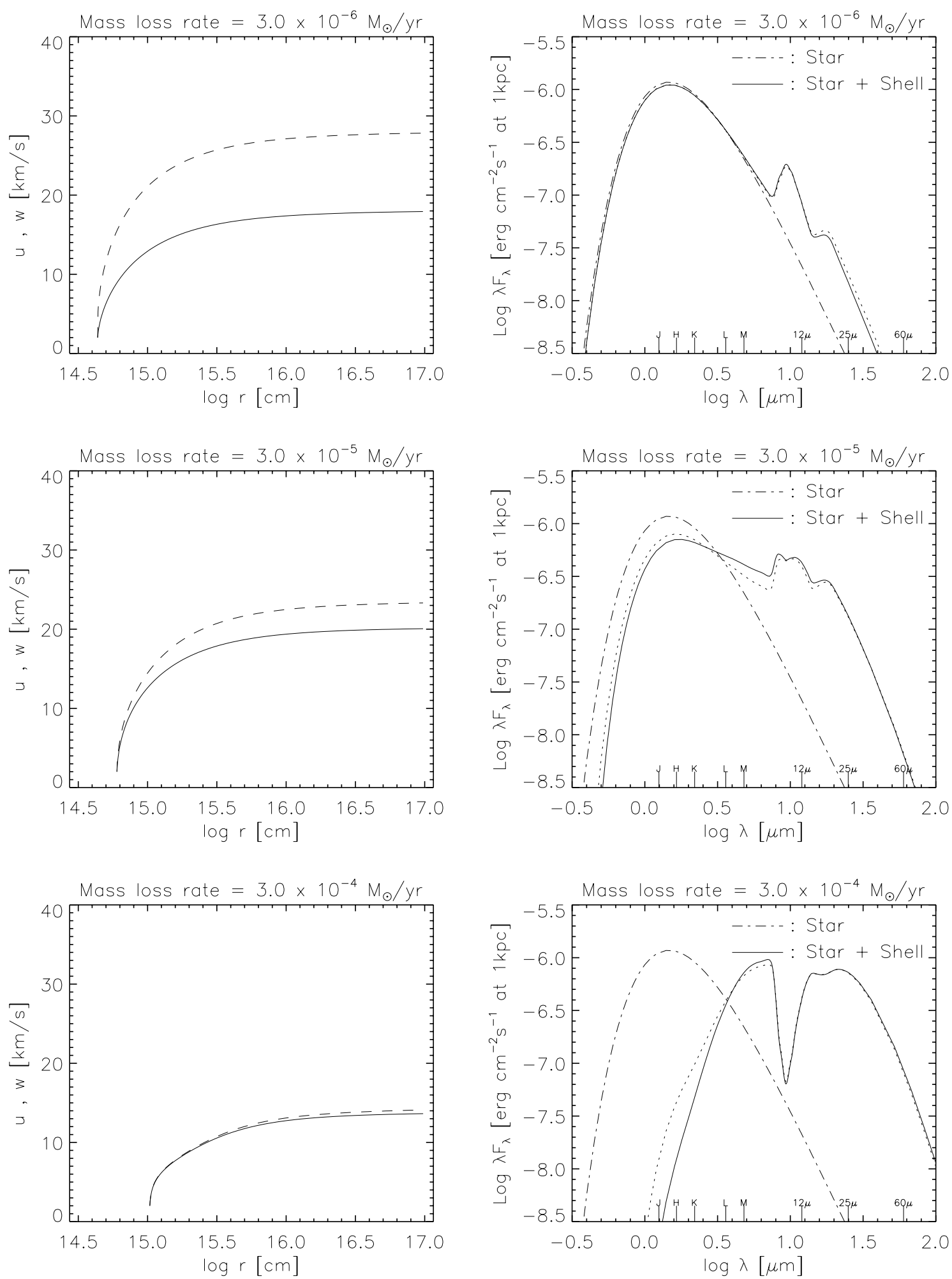

Fig. 3. Same as Fig. 2 but for an Oxygen star with $M_{*}=4 M_{\odot}, L_{*}=5.010^{4} L_{\odot}$, and $T_{\text {eff }}=2500 \mathrm{~K}$ (note that the range of mass loss rates and the scaling of the plots is different). As in the previous sequence (Fig. 2) the initial velocity at the dust formation point is $u_{1}=2 \mathrm{~km} / \mathrm{s}$. More information about these models (D5_m, D3_m, D1_m) and additional runs of this sequence is listed in Table 3. The dotted spectral energy distribution was computed from a simplified model, assuming a constant velocity, $u=w=u_{\mathrm{e}}$, where $u_{\mathrm{e}}$ is the terminal gas outflow velocity obtained from the hydrodynamical model with the same parameters 
Stellar parameters: $M_{*}=10 \mathrm{M}_{\odot}, T_{\text {eff }}=2500 \mathrm{~K}, L_{*}=4 \times 10^{5}$; Dust: astronomical silicates, $a=0.05 \mu m, \delta=0.005$
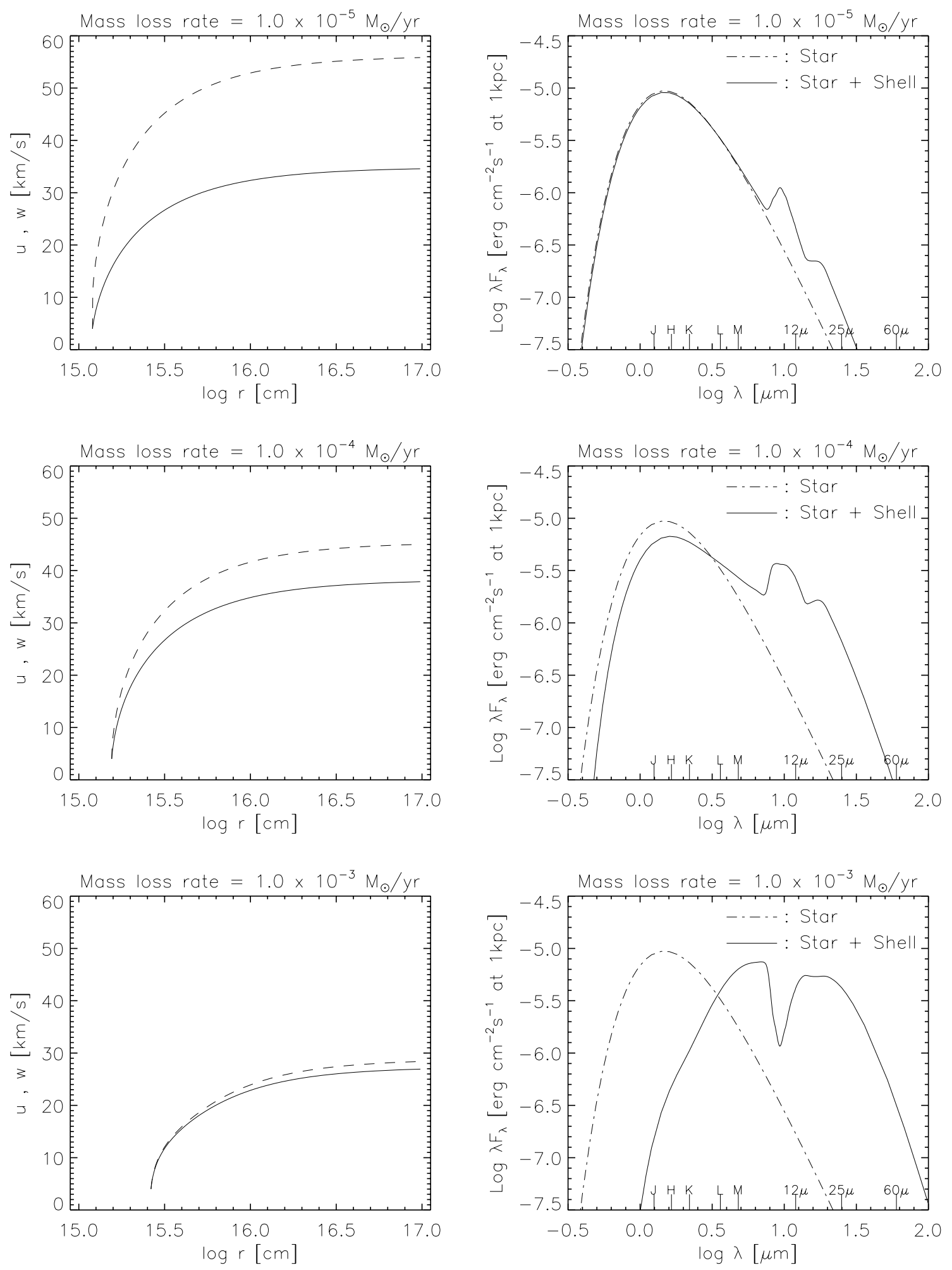

Fig. 4. Same as Fig. 2 but for an Oxygen star with $M_{*}=10 M_{\odot}, L_{*}=4.010^{5} L_{\odot}$, and $T_{\text {eff }}=2500 \mathrm{~K}$. The initial velocity at the dust formation point is $u_{1}=4 \mathrm{~km} / \mathrm{s}$. More information about these models (E5_m, E3_m, E1_m) and additional runs of this sequence is listed in Table 4 
Stellar parameters: $M_{*}=30 M_{\odot}, T_{\text {eff }}=2500 \mathrm{~K}, L_{*}=4 \times 10^{5}$; Dust: astronomical silicates, $a=0.05 \mu \mathrm{m}, \delta=0.005$
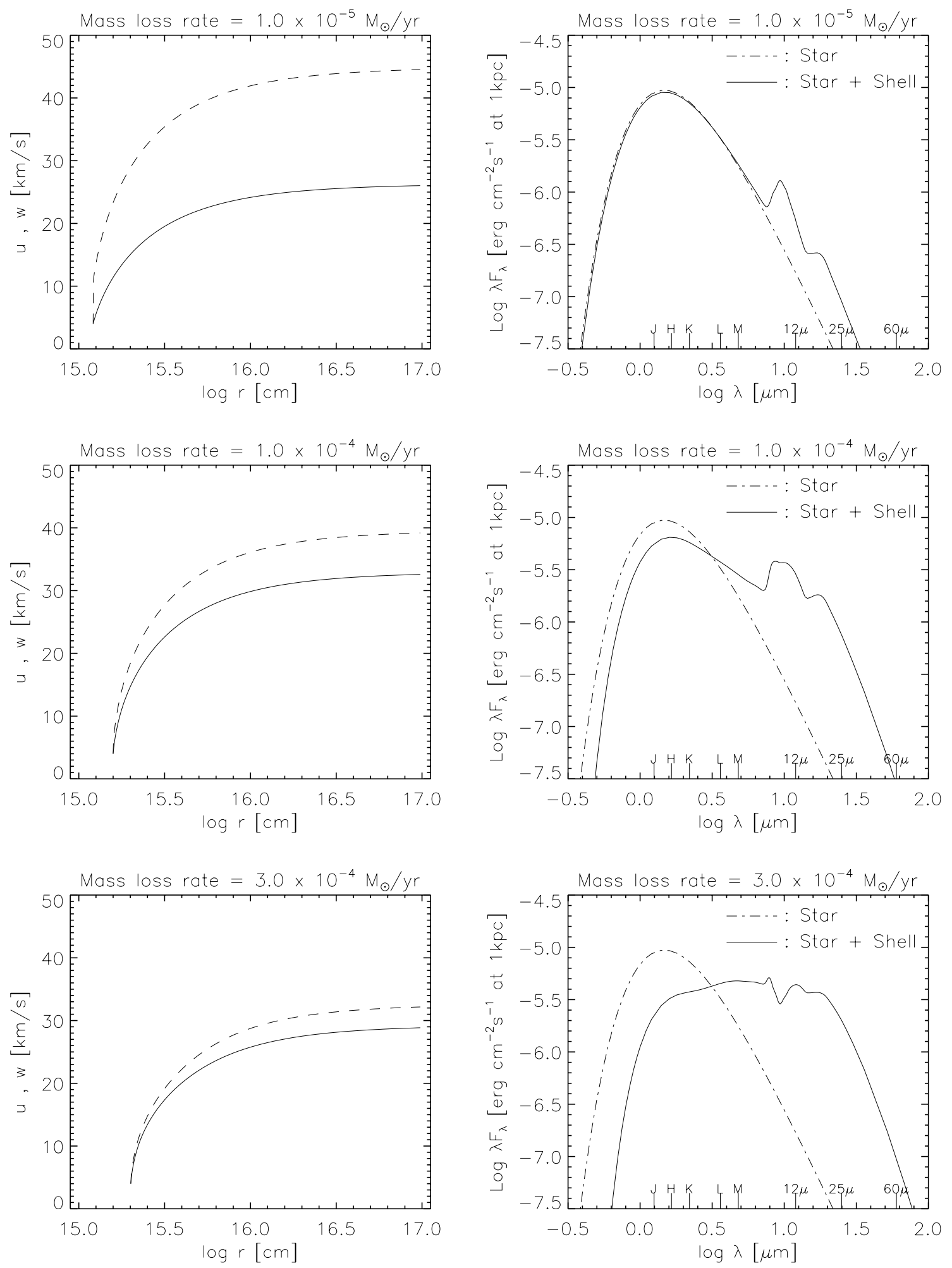

Fig. 5. Same as Fig. 2 but for an Oxygen star with $M_{*}=30 M_{\odot}, L_{*}=4.010^{5} L_{\odot}$, and $T_{\text {eff }}=2500 \mathrm{~K}$. As in the previous sequence (Fig. 4) the initial velocity at the dust formation point is $u_{1}=4 \mathrm{~km} / \mathrm{s}$. More information about these models (F4_m, F2_m, F1_m) and additional runs of this sequence is listed in Table 5 

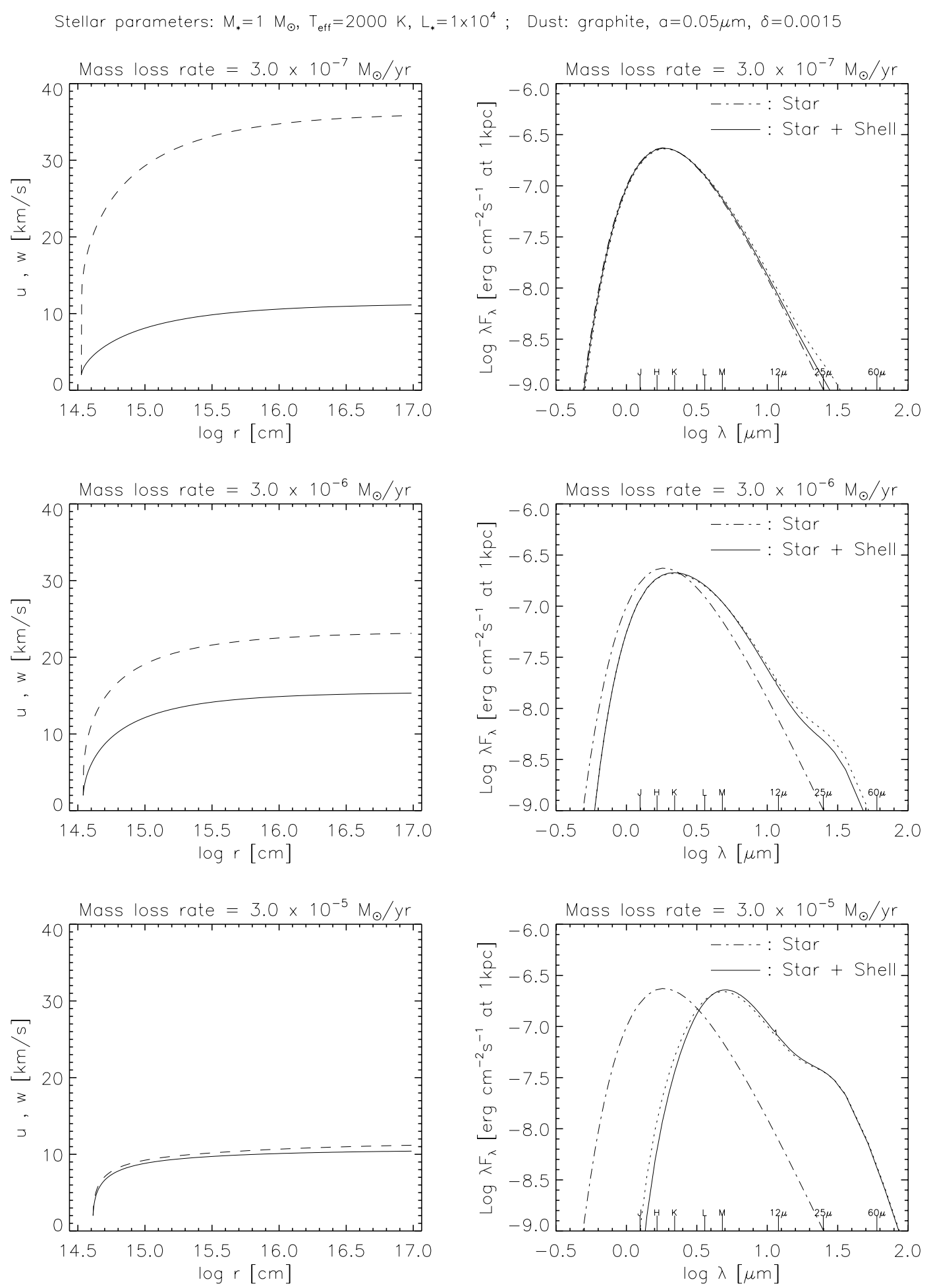

Fig. 6. Model results for a Carbon star with $M_{*}=1 M_{\odot}, L_{*}=1.010^{4} L_{\odot}$, and $T_{\text {eff }}=2000 \mathrm{~K}$ for 3 different mass loss rates (indicated in the heading of each panel). The dust is assumed to consist of grains of graphite with properties given in Table 1. The initial velocity at the dust formation point is assumed to be $u_{1}=2 \mathrm{~km} / \mathrm{s}$. The left hand panels show the gas velocity (solid) and the dust velocity (dashed) as a function of radial distance. The right hand panels show the corresponding stellar input spectrum (dot-dashed) and the emergent spectral energy distribution (solid) which is the result of processing of the stellar radiation by the dusty envelope. More information about these models (G6_m, G3_m, G1_m) and additional runs of this sequence is listed in Table 6. The dotted spectral energy distribution was computed from a simplified model, assuming a constant velocity, $u=w=u_{\mathrm{e}}$, where $u_{\mathrm{e}}$ is the terminal gas outflow velocity obtained from the hydrodynamical model with the same parameters 
Stellar parameters: $M_{*}=3 M_{\odot}, T_{\text {eff }}=2000 \mathrm{~K}, L_{*}=5 \times 10^{4}$; Dust: graphite, $a=0.05 \mu \mathrm{m}, \delta=0.0015$
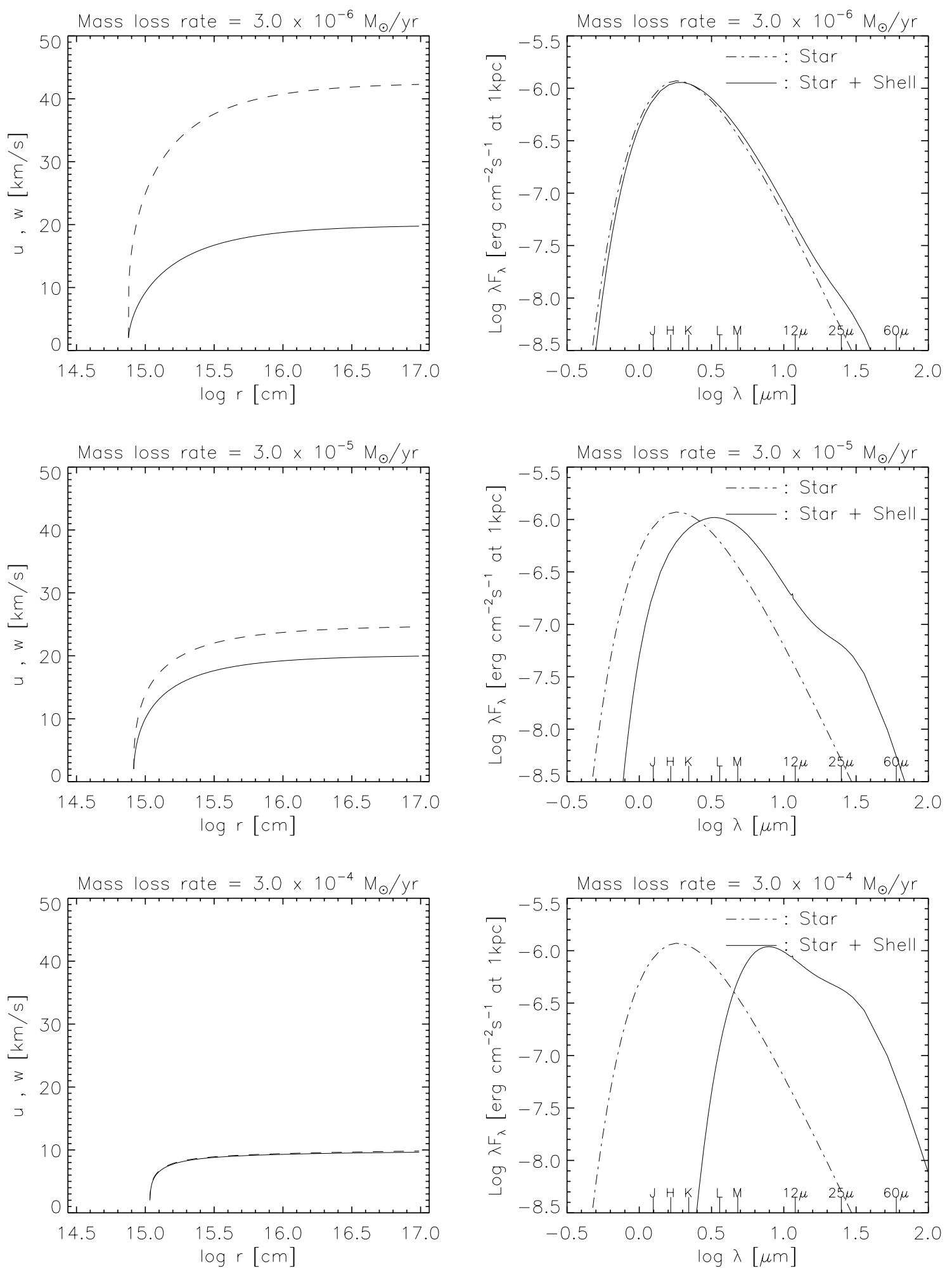

Fig. 7. Same as Fig. 6 but for a Carbon star with $M_{*}=3 M_{\odot}, L_{*}=5.010^{4} L_{\odot}$, and $T_{\text {eff }}=2000 \mathrm{~K}$ (note that the range of mass loss rates and the scaling of the plots is different). More information about these models (H6_m, H4_m, H2_m) and additional runs of this sequence is listed in Table 7 
Stellar parameters: $M_{*}=5 M_{\odot}, T_{\text {eff }}=2000 \mathrm{~K}, L_{*}=5 \times 10^{4}$; Dust: graphite, $a=0.05 \mu \mathrm{m}, \delta=0.0015$
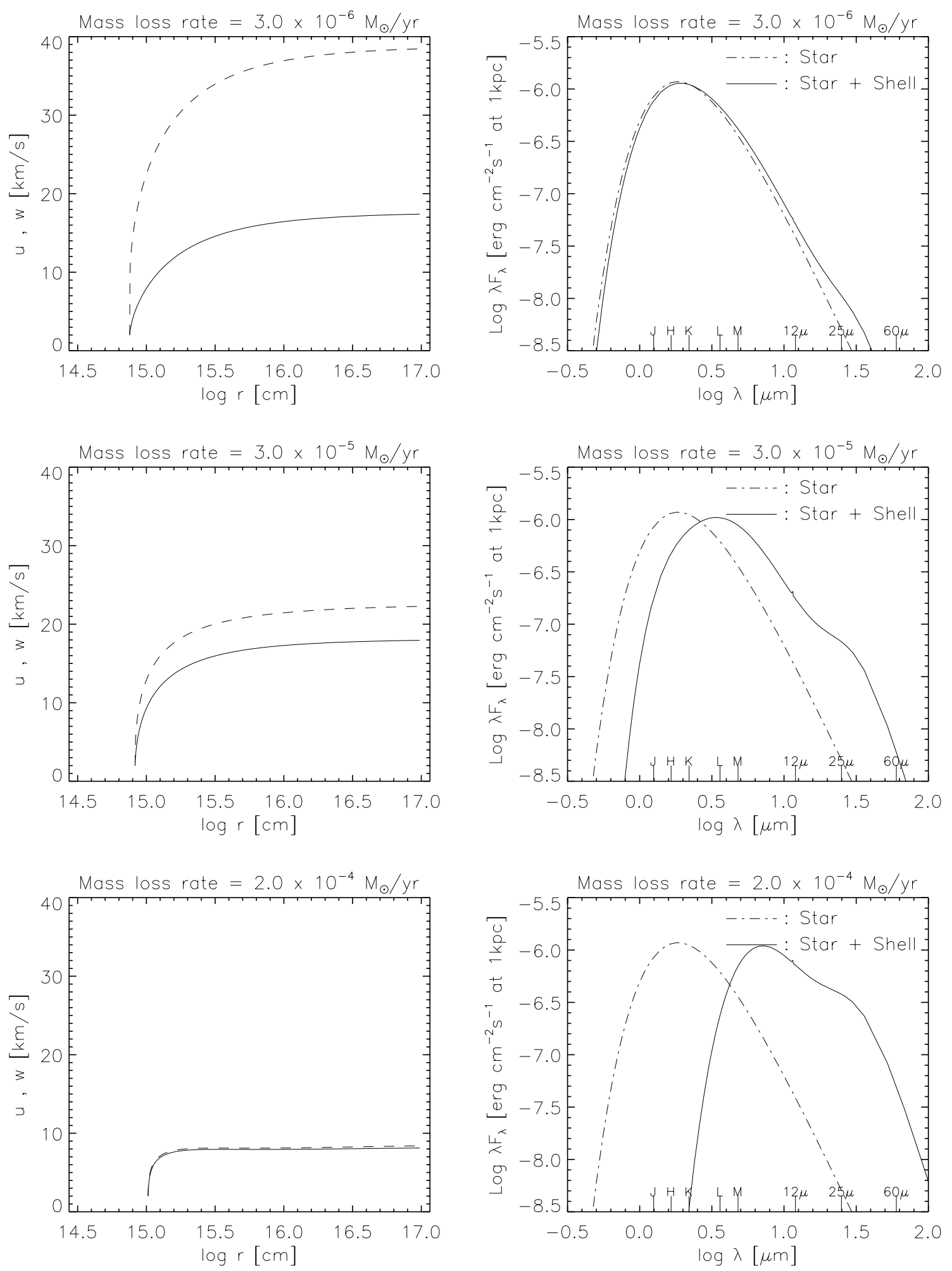

Fig. 8. Same as Fig. 6 but for a Carbon star with $M_{*}=5 M_{\odot}, L_{*}=5.010^{4} L_{\odot}$, and $T_{\text {eff }}=2000 \mathrm{~K}$. More information about these models (I5_m, I3_m, I1_m) and additional runs of this sequence is listed in Table 8 
Stellar parameters: $M_{*}=1 M_{\odot}, T_{\text {eff }}=2000 \mathrm{~K}, L_{*}=1 \times 10^{4}$; Dust: amorphous carbon, $a=0.05 \mu \mathrm{m}, \delta=0.0015$
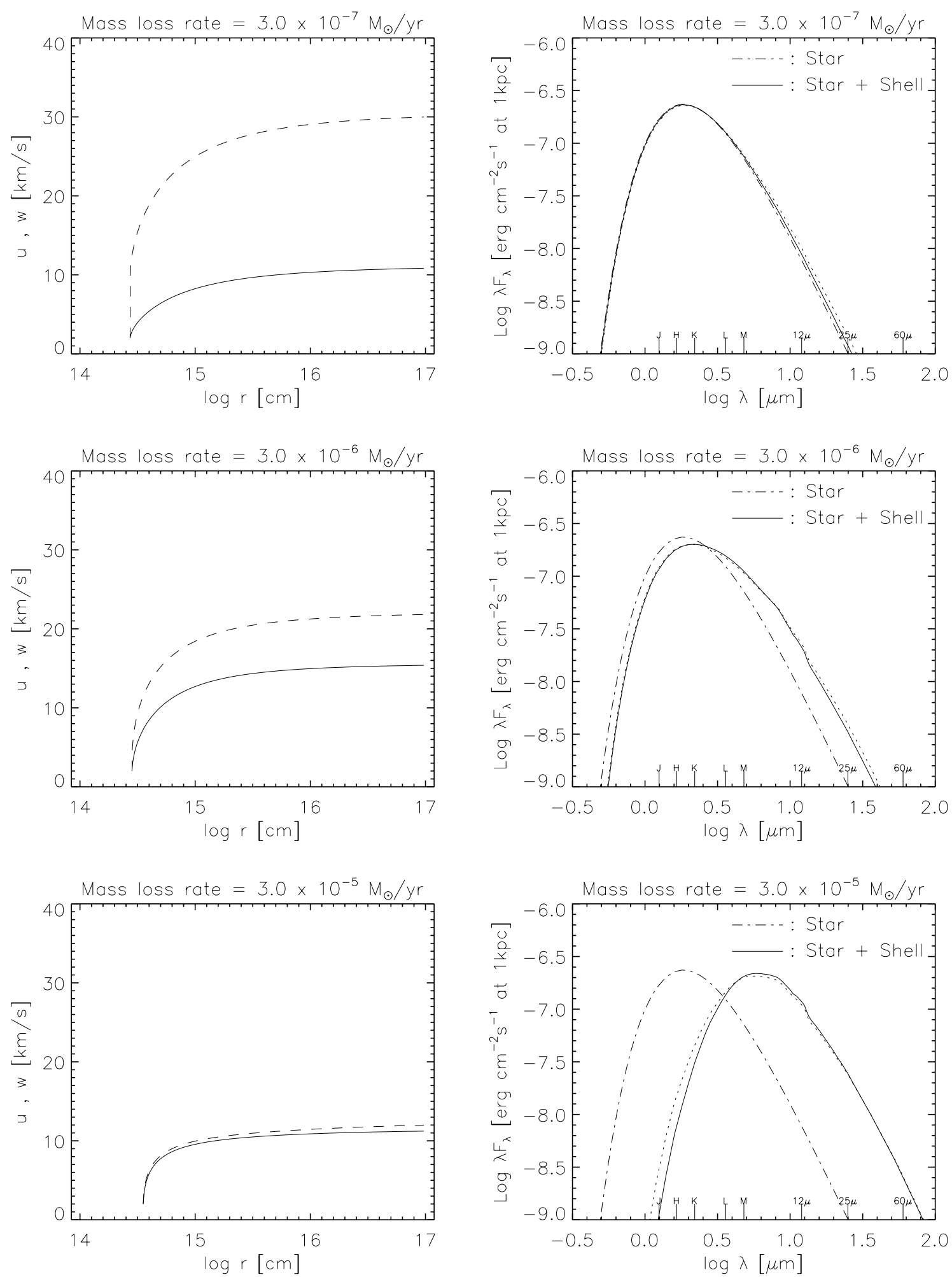

Fig. 9. Same as Fig. 6 but for dust consisting of grains of amorphous carbon with properties given in Table 1. More information about these models (J6_m, J3_m, J1_m) and additional runs of this sequence is listed in Table 9. The dotted spectral energy distribution was computed from a simplified model, assuming a constant velocity, $u=w=u_{\mathrm{e}}$, where $u_{\mathrm{e}}$ is the terminal gas outflow velocity obtained from the hydrodynamical model with the same parameters 
Stellar parameters: $M_{*}=3 M_{\odot}, T_{\text {eff }}=2000 \mathrm{~K}, L_{*}=5 \times 10^{4}$; Dust: amorphous carbon, $a=0.05 \mu \mathrm{m}, \delta=0.0015$
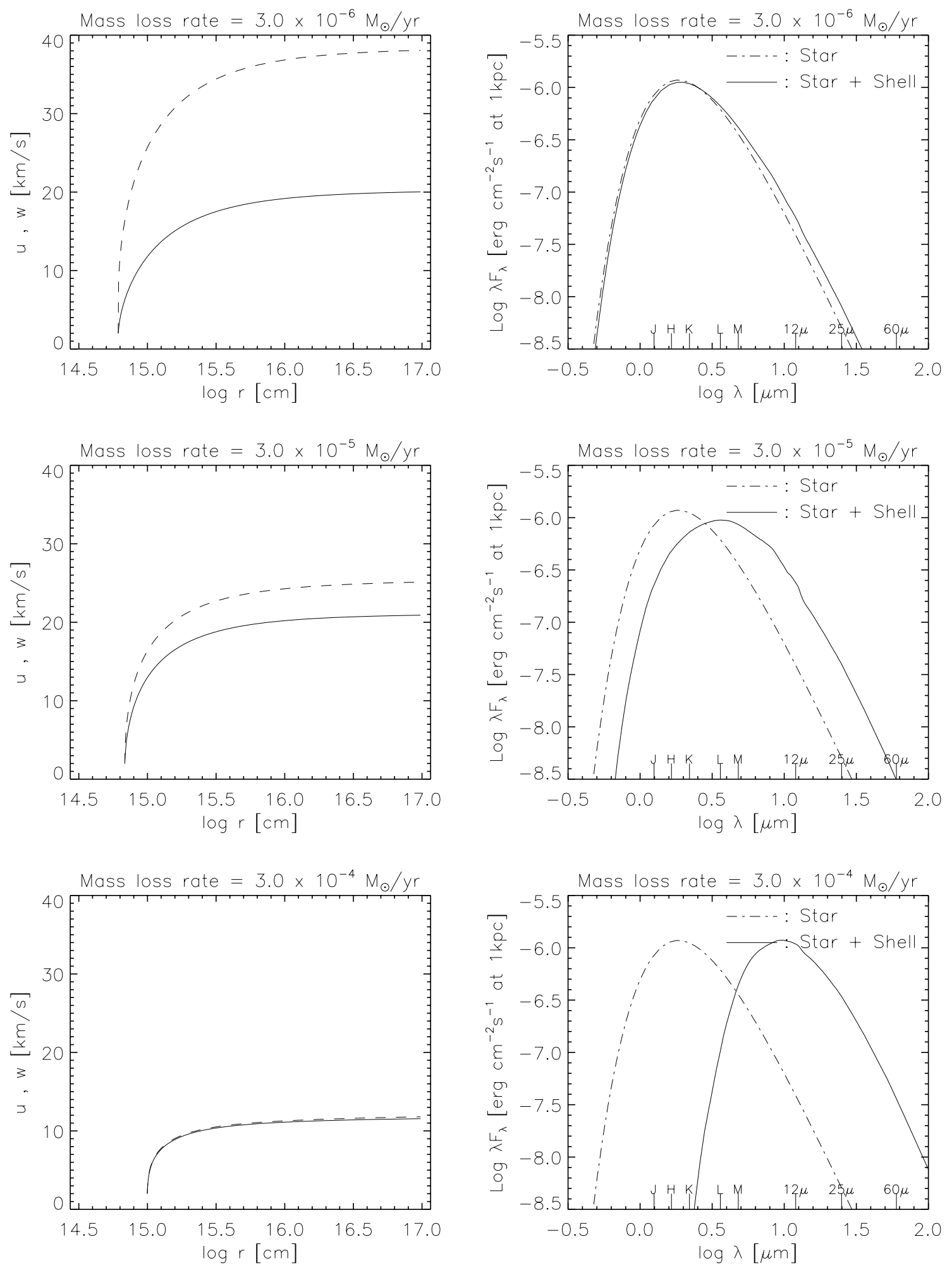

Fig. 10. Same as Fig. 9 but for a Carbon star with $M_{*}=3 M_{\odot}, L_{*}=5.010^{4} L_{\odot}$, and $T_{\text {eff }}=2000 \mathrm{~K}$ (note that the range of mass loss rates and the scaling of the plots is different). More information about these models (K6_m, K4_m, K2_m) and additional runs of this sequence is listed in Table 10 
Stellar parameters: $M_{*}=5 M_{\odot}, T_{\text {eff }}=2000 \mathrm{~K}, L_{*}=5 \times 10^{4}$; Dust: amorphous carbon, $a=0.05 \mu \mathrm{m}, \delta=0.0015$
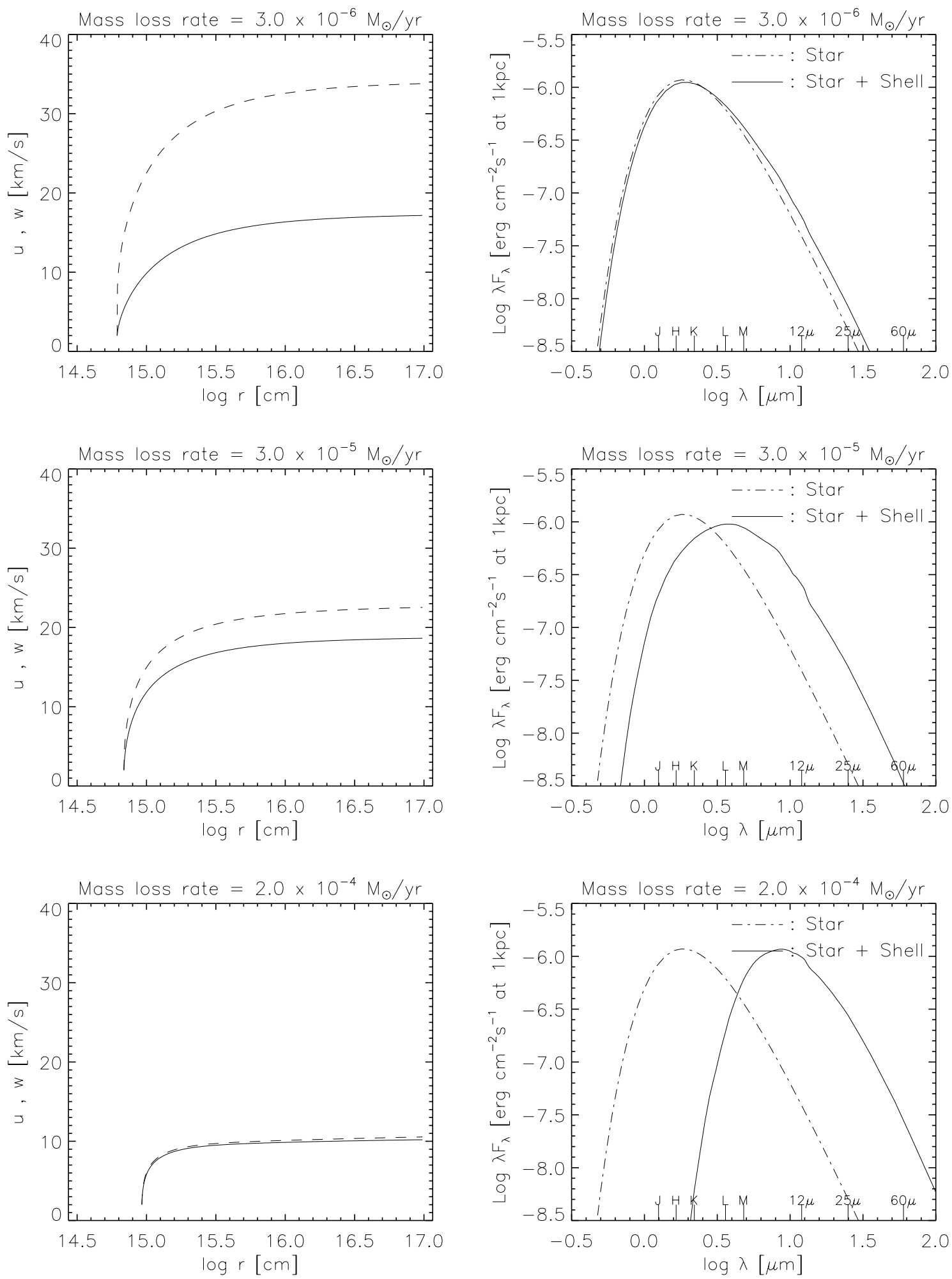

Fig. 11. Same as Fig. 9 but for a Carbon star with $M_{*}=5 M_{\odot}, L_{*}=5.010^{4} L_{\odot}$, and $T_{\text {eff }}=2000 \mathrm{~K}$. More information about these models (L5_m, L3_m, L1_m) and additional runs of this sequence is listed in Table 11 

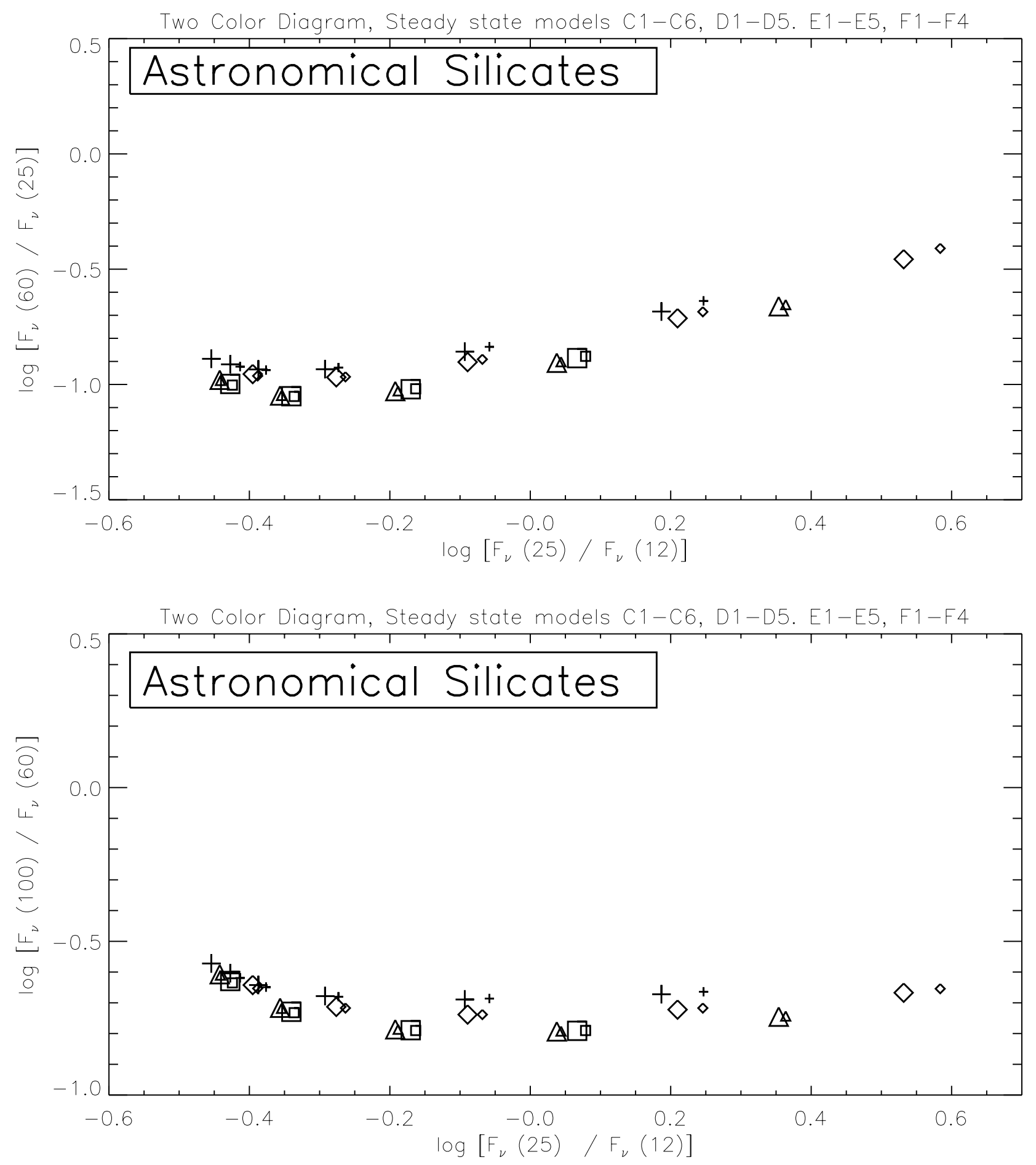

Fig. 12. Top: IRAS two-color diagram for the Oxygen star models $C(+)$, D $(\diamond), \mathrm{E}(\triangle)$ and F ( $\square)$. For each large symbol, indicating the position of a model computed with gas pressure, there is a corresponding small symbol close to it, showing the position of the respective model computed without gas pressure. Bottom: Same as above, but showing the flux ratio $F_{\nu}(100 \mu \mathrm{m}) / F_{\nu}(60 \mu \mathrm{m})$ in the ordinate 


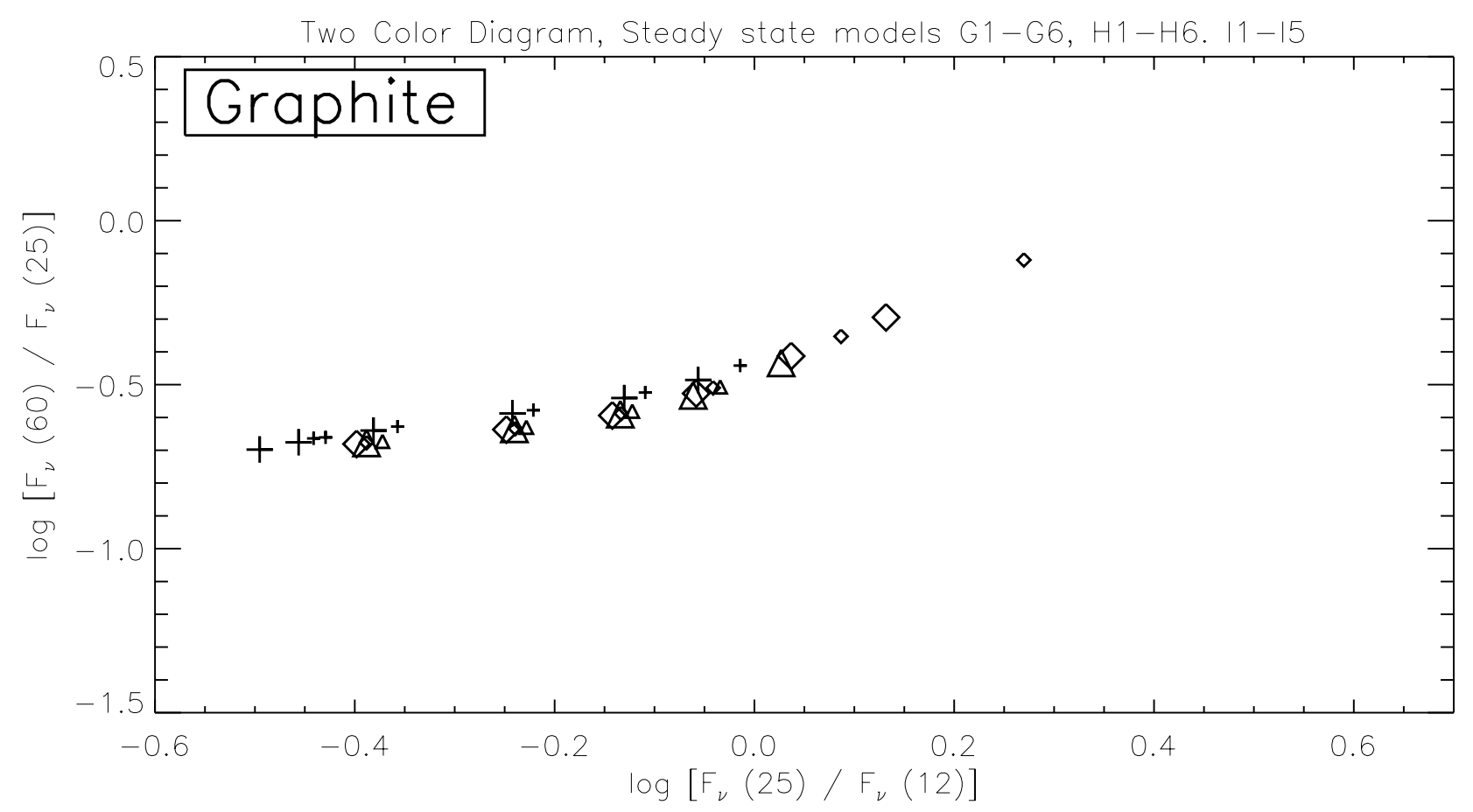

Two Color Diagram, Steady state models G1-G6, H1-H6. $11-15$

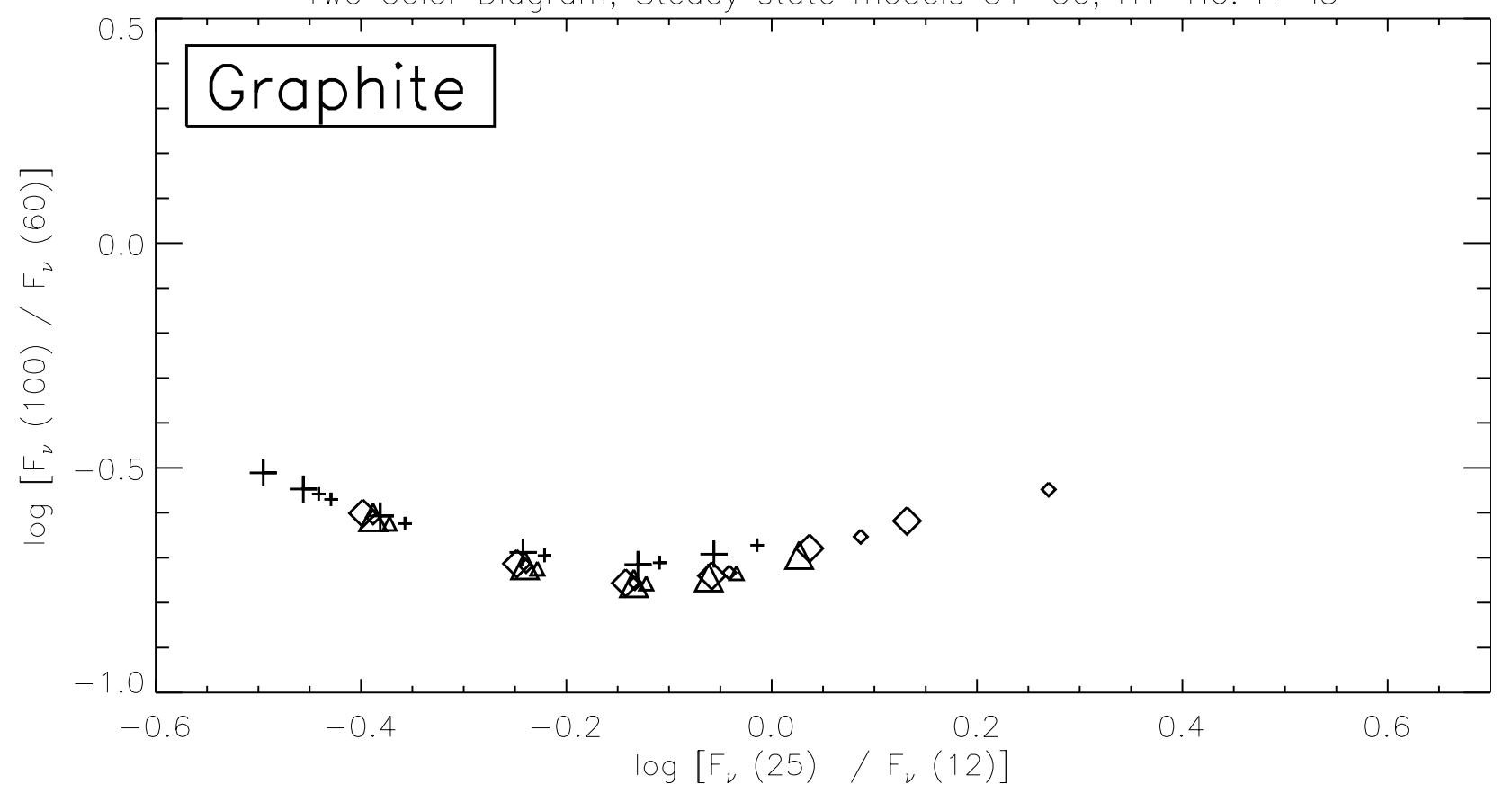

Fig. 13. Top: IRAS two-color diagram for the Carbon star models $G(+), H(\diamond)$, and $I(\triangle)$, based on dust grains consisting of graphite. For each large symbol, indicating the position of a model computed with gas pressure, there is a corresponding small symbol close to it, showing the position of the respective model computed without gas pressure. Bottom: Same as above, but showing the flux ratio $F_{\nu}(100 \mu \mathrm{m}) / F_{\nu}(60 \mu \mathrm{m})$ in the ordinate 

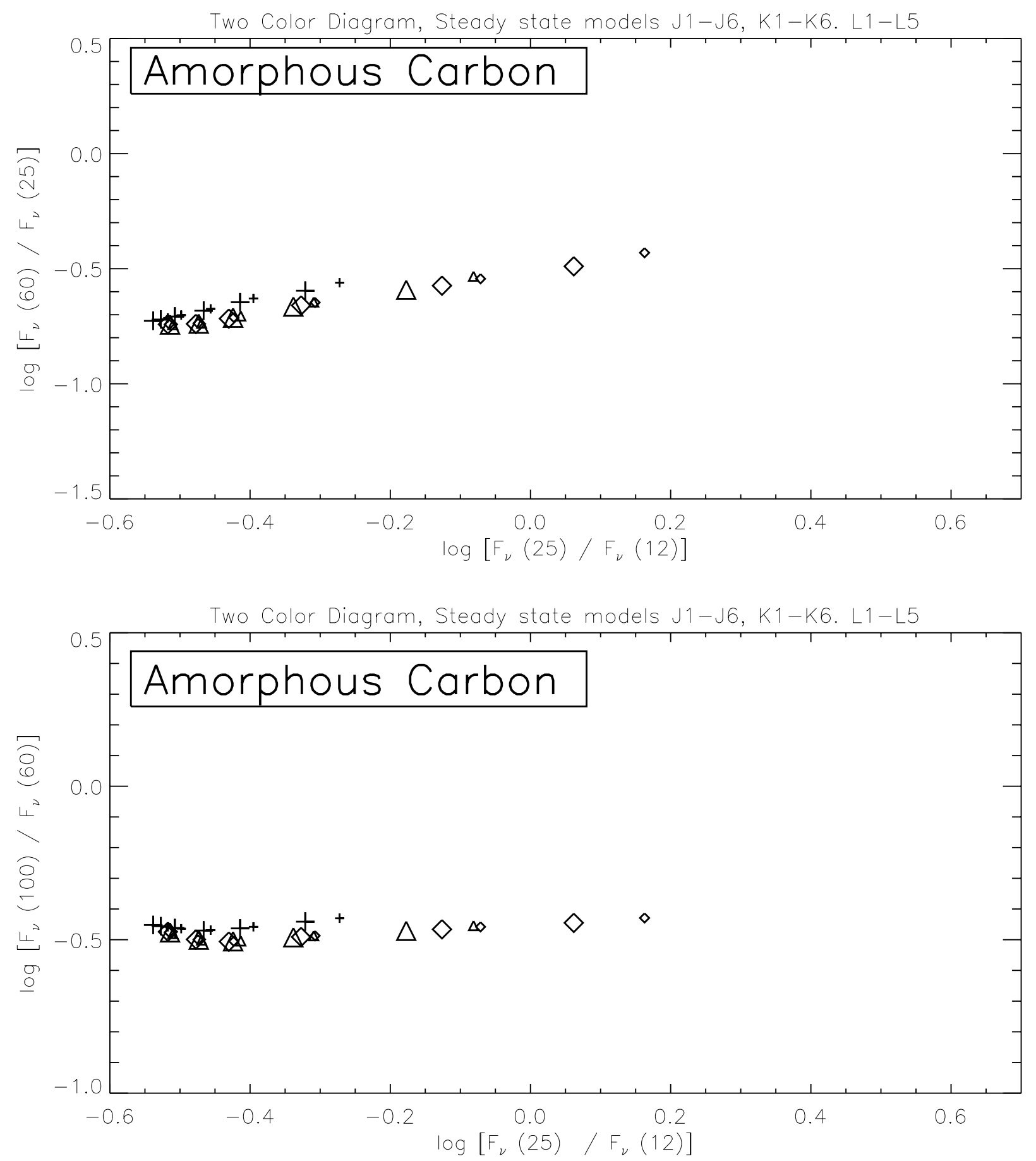

Fig. 14. Top: IRAS two-color diagram for the Carbon star models $J(+), \mathrm{K}(\diamond)$, and $\mathrm{L}(\triangle)$, based on dust grains consisting of amorphous carbon. For each large symbol, indicating the position of a model computed with gas pressure, there is a corresponding small symbol close to it, showing the position of the respective model computed without gas pressure. Bottom: Same as above, but showing the flux ratio $F_{\nu}(100 \mu \mathrm{m}) / F_{\nu}(60 \mu \mathrm{m})$ in the ordinate 

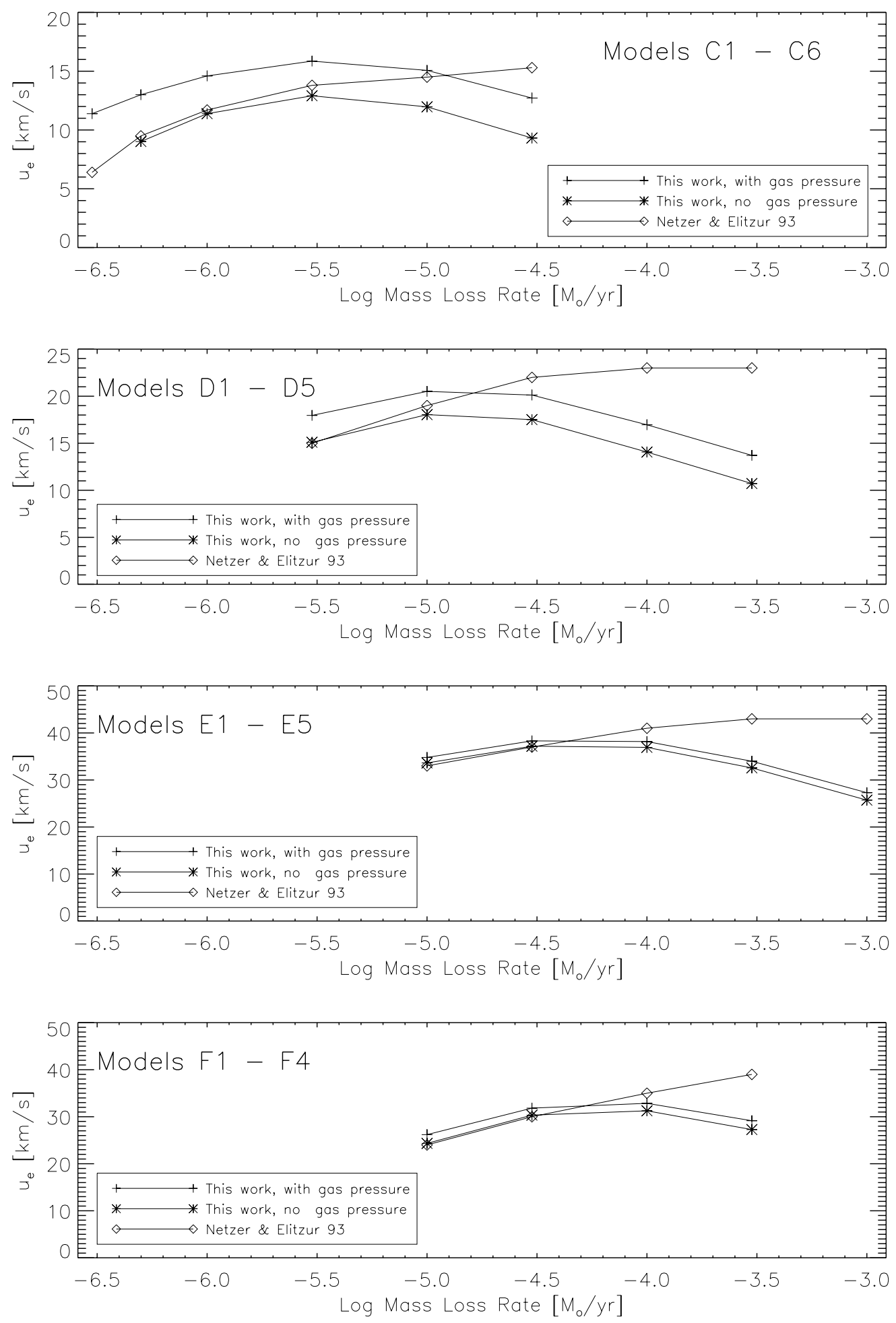

Fig. 15. Terminal gas velocity, $u_{\mathrm{e}}$, for different Oxygen stars obtained with our radiation hydrodynamics code including $(+)$ and ignoring $(*)$ gas pressure in the equations of motion compared with the data given in Table 3 of NE $(\diamond)$ for different mass loss rates. Note that NE ignore gas pressure. The dust is assumed to consist of grains of astronomical silicates with $a=0.05 \mu \mathrm{m}$, $\rho_{\mathrm{i}}=3.3 \mathrm{~g} \mathrm{~cm}^{-3}, \delta=0.005, T_{\text {cond }}=800 \mathrm{~K}$. From top to bottom, the parameters are: $M_{*}=1 M_{\odot}, L_{*}=1.010^{4} L_{\odot}$, $T_{\text {eff }}=3000 \mathrm{~K}$ and $u_{1}=2 \mathrm{~km} / \mathrm{s}$ (C models); $M_{*}=4 M_{\odot}, L_{*}=5.010^{4} L_{\odot}, T_{\text {eff }}=2500 \mathrm{~K}$ and $u_{1}=2 \mathrm{~km} / \mathrm{s} \quad(\mathrm{D}$ models $) ;$ $M_{*}=10 M_{\odot}, L_{*}=4.010^{5} L_{\odot}, T_{\text {eff }}=2500 \mathrm{~K}$ and $u_{1}=4 \mathrm{~km} / \mathrm{s}$ (E models); $M_{*}=30 M_{\odot}, L_{*}=4.010^{5} L_{\odot}, T_{\text {eff }}=2500 \mathrm{~K}$ and $u_{1}=4 \mathrm{~km} / \mathrm{s}$ (F models) 

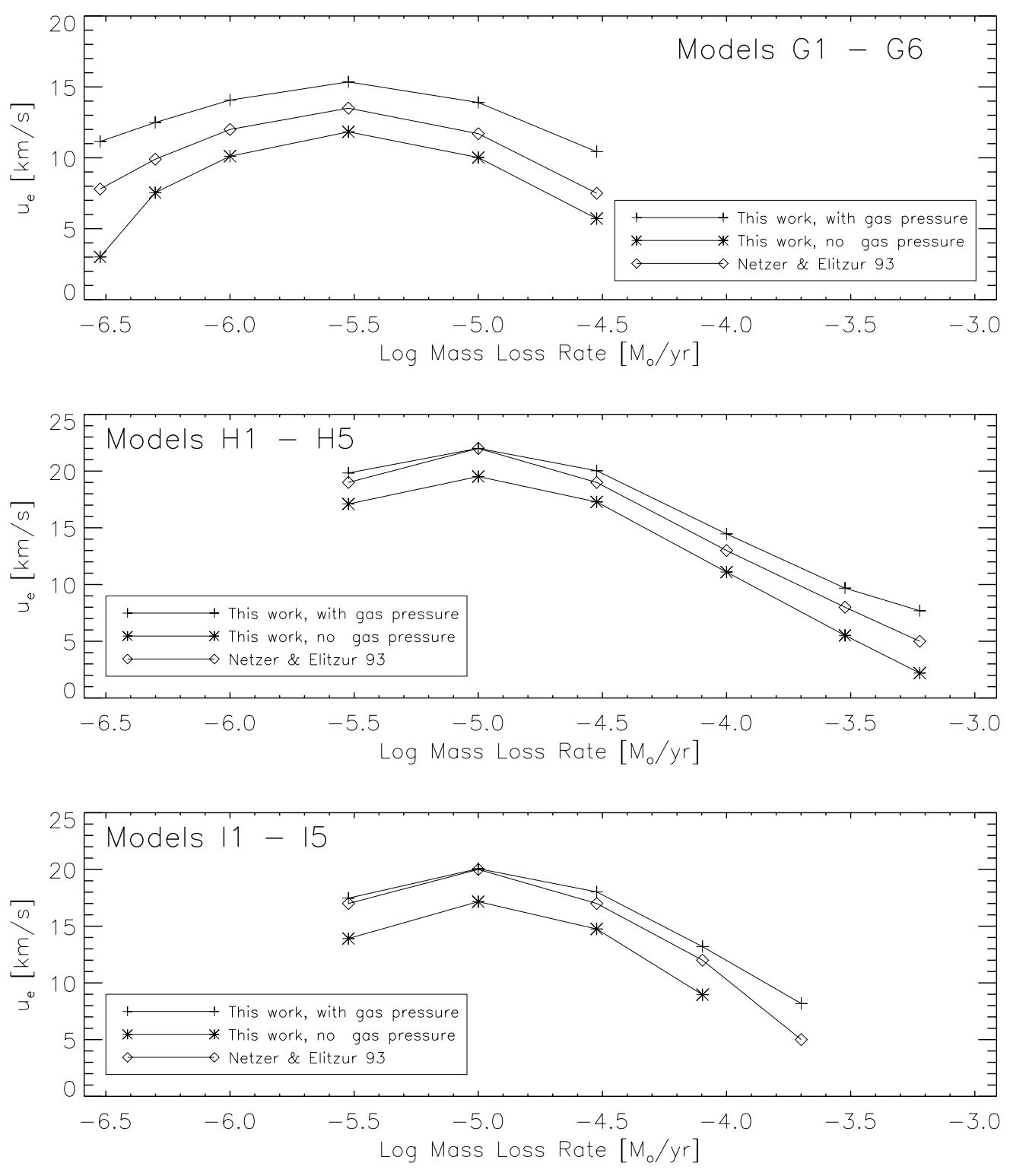

Fig. 16. Terminal gas velocity, $u_{\mathrm{e}}$, for different Carbon stars obtained with our radiation hydrodynamics code including $(+)$ and ignoring $(*)$ gas pressure in the equations of motion compared with the data given in Table 3 of NE ( $\diamond)$ for different mass loss rates. Note that NE ignore gas pressure. The dust is assumed to consist of grains of graphite with $a=0.05 \mu \mathrm{m}$, $\rho_{\mathrm{i}}=2.26 \mathrm{~g} \mathrm{~cm}^{-3}, \delta=0.0015, T_{\text {cond }}=850 \mathrm{~K}$. From top to bottom, the parameters are: $M_{*}=1 M_{\odot}, L_{*}=1.010^{4} L_{\odot}$, $T_{\text {eff }}=2000 \mathrm{~K}$ and $u_{1}=2 \mathrm{~km} / \mathrm{s}$ (G models); $M_{*}=3 M_{\odot}, L_{*}=5.010^{4} L_{\odot}, T_{\text {eff }}=2000 \mathrm{~K}$ and $u_{1}=2 \mathrm{~km} / \mathrm{s}$ (H models); $M_{*}=5 M_{\odot}, L_{*}=5.010^{4} L_{\odot}, T_{\text {eff }}=2000 \mathrm{~K}$ and $u_{1}=2 \mathrm{~km} / \mathrm{s}$ (I models) 

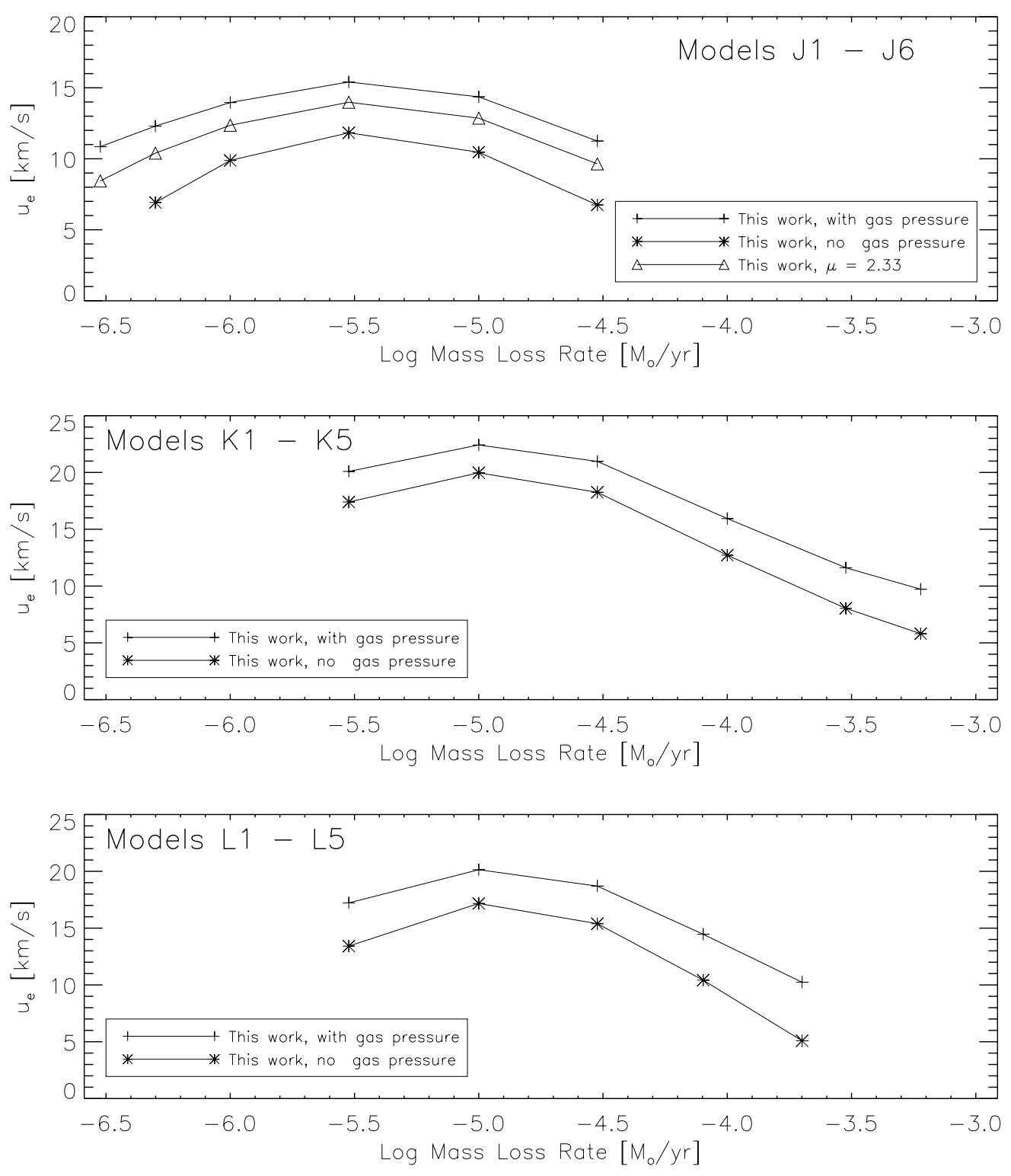

Fig. 17. Terminal gas velocity, $u_{\mathrm{e}}$, for different Carbon stars obtained with our radiation hydrodynamics code including $(+)$ and ignoring $(*)$ gas pressure in the equations of motion. The dust is assumed to consist of grains of amorphous carbon with $a=0.05 \mu \mathrm{m}, \rho_{\mathrm{i}}=1.85 \mathrm{~g} \mathrm{~cm}^{-3}, \delta=0.0015, T_{\text {cond }}=850 \mathrm{~K}$. For models J, triangles indicate the $u_{\mathrm{e}}$ resulting when the gas pressure terms are computed with a molecular weight $\mu=2.33$ (molecular hydrogen) instead of the standard assumption $\mu \approx 1.3$ (atomic hydrogen). From top to bottom, the parameters are: $M_{*}=1 M_{\odot}, L_{*}=1.010^{4} L_{\odot}, T_{\text {eff }}=2000 \mathrm{~K}$ and $u_{1}=2 \mathrm{~km} / \mathrm{s}(\mathrm{J}$ models); $M_{*}=3 M_{\odot}, L_{*}=5.010^{4} L_{\odot}, T_{\text {eff }}=2000 \mathrm{~K}$ and $u_{1}=2 \mathrm{~km} / \mathrm{s}$ (K models); $M_{*}=5 M_{\odot}, L_{*}=5.010^{4} L_{\odot}$, $T_{\text {eff }}=2000 \mathrm{~K}$ and $u_{1}=2 \mathrm{~km} / \mathrm{s}$ (L models) 

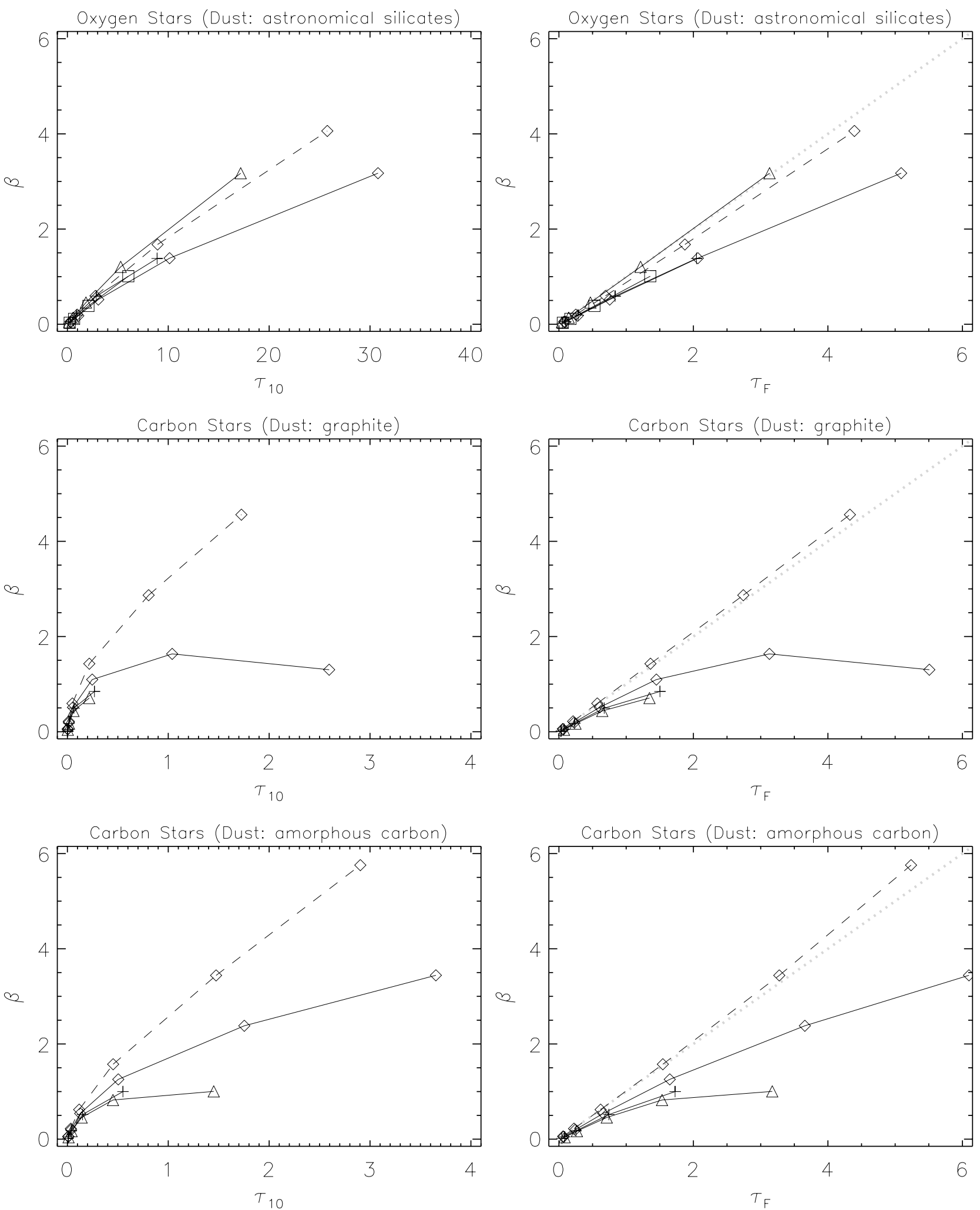

Fig. 18. Coupling factor $\beta=\dot{M} u_{\mathrm{e}} c / L_{*}$ as a function of $\tau_{10}$, (optical depth at $\lambda 10 \mu \mathrm{m}$ ) and $\tau_{\mathrm{F}}$ (flux-averaged optical depth; cf. Eq. 25). Top frames: Results for our Oxygen star models computed without gas pressure (Ci_n $(+)$; Di_n $(\diamond)$; Ei_n $(\triangle)$; Fi_n $(\square))$ and for models Di_m $(\diamond--\diamond)$ computed with gas pressure. Middle frames : Results for our Carbon star models with graphite dust, computed without gas pressure (Gi_n $(+)$; Hi_n $(\diamond)$; Ii_n $(\triangle))$ and for models Hi_m $(\diamond--\diamond)$ computed with gas pressure. Bottom frames: Results for our Carbon star models with amorphous carbon dust, computed without gas pressure $($ Ji_n $(+)$; Ki_n $(\diamond)$; Li_n $(\triangle))$ and for models Ki_m $(\diamond--\diamond)$ computed with gas pressure. The dotted diagonal in the right-hand frames indicates the relation $\beta=\tau_{\mathrm{F}}$, the maximum slope attainable by high luminosity models without gas pressure 